\title{
Second order superintegrable systems in conformally flat spaces. IV. The classical 3D Stäckel transform and 3D classification theory
}

\author{
E. G. Kalnins \\ Department of Mathematics and Statistics, University of Waikato, Hamilton, \\ New Zealand \\ J. M. Kress ${ }^{\mathrm{a})}$ \\ School of Mathematics, The University of New South Wales, Sydney NSW 2052, Australia \\ W. Miller, Jr. \\ School of Mathematics, University of Minnesota, Minneapolis, Minnesota 55455
}

(Received 14 January 2006; accepted 10 March 2006; published online 27 April 2006)

\begin{abstract}
This article is one of a series that lays the groundwork for a structure and classification theory of second order superintegrable systems, both classical and quantum, in conformally flat spaces. In the first part of the article we study the Stäckel transform (or coupling constant metamorphosis) as an invertible mapping between classical superintegrable systems on different three-dimensional spaces. We show first that all superintegrable systems with nondegenerate potentials are multiseparable and then that each such system on any conformally flat space is Stäckel equivalent to a system on a constant curvature space. In the second part of the article we classify all the superintegrable systems that admit separation in generic coordinates. We find that there are eight families of these systems. (C) 2006 American Institute of Physics. [DOI: 10.1063/1.2191789]
\end{abstract}

\section{INTRODUCTION}

This is a continuation of the series ${ }^{1-3}$ whose purpose is to lay the groundwork for a structure and classification theory of second order superintegrable systems, both classical and quantum, in complex conformally flat spaces. Real spaces are considered as restrictions of these to the various real forms. In Refs. 1 and 3 we have given examples in two and three dimensions, described the background as well as the interest and importance of these systems in mathematical physics and given many applications relevant to such systems on conformally flat spaces. Observed features of the systems are multiseparability, closure of the quadratic algebra of second order symmetries at order 6, use of representation theory of the quadratic algebra to derive spectral properties of the quantum Schrödinger operator, and a close relationship with exactly solvable and quasiexactly solvable problems. Our approach is, rather than focus on particular spaces and systems, to use a general theoretical method based on integrability conditions to derive structure common to all systems. In distinction to the two-dimensional (2D) case, there are relatively few papers considering superintegrability on spaces of dimension $\geqslant 3$. A few exceptions are Refs. 4-13. Except for our own work, no one appears to have studied the detailed structure and classification theory for these higher dimensional systems.

In the first part of this article we study the Stäckel transform, or coupling constant metamorphosis, ${ }^{14,15}$ for three-dimensional (3D) classical superintegrable systems. Recall that for a classical 3D system on a conformally flat space we can always choose local coordinates $x, y, z$, not unique, such that the Hamiltonian takes the form $H=\left(p_{1}^{2}+p_{2}^{2}+p_{3}^{2}\right) / \lambda(x, y, z)+V(x, y, z)$. This

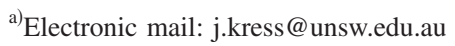


system is second order superintegrable with nondegenerate potential $V=V(x, y, z, \alpha, \beta, \gamma, \delta)$ if it admits five functionally independent quadratic constants of the motion (i.e., generalized symmetries) $S_{k}=\sum_{i j} a_{(k)}^{i j} p_{i} p_{j}+W_{(k)}(x, y, \alpha, \beta, \gamma)$. As described in Ref. 3 , the potential $V$ is nondegenerate if it satisfies a system of coupled partial differential equations of the form

$$
\begin{gathered}
V_{22}=V_{11}+A^{22} V_{1}+B^{22} V_{2}+C^{22} V_{3}, \quad V_{33}=V_{11}+A^{33} V_{1}+B^{33} V_{2}+C^{33} V_{3}, \\
V_{12}=A^{12} V_{1}+B^{12} V_{2}+C^{12} V_{3}, \quad V_{13}=A^{13} V_{1}+B^{13} V_{2}+C^{13} V_{3}, \\
V_{23}=A^{23} V_{1}+B^{23} V_{2}+C^{23} V_{3},
\end{gathered}
$$

whose integrability conditions are satisfied identically. The analytic functions $A^{i j}, B^{i j}, C^{i j}$ are determined uniquely from the Bertrand-Darboux equations for the five constants of the motion and are analytic except for a finite number of poles. At any regular point $\mathbf{x}_{0}=\left(x_{0}, y_{0}, z_{0}\right)$, i.e., a point where the $A^{i j}, B^{i j}$, and $C^{i j}$ are defined and analytic and the constants of the motion are functionally independent, we can prescribe the values of $V\left(\mathbf{x}_{0}\right), V_{1}\left(\mathbf{x}_{0}\right), V_{2}\left(\mathbf{x}_{0}\right), V_{3}\left(\mathbf{x}_{0}\right), V_{11}\left(\mathbf{x}_{0}\right)$ arbitrarily and obtain a unique solution of (1). Here, $V_{1}=\partial V / \partial x, V_{2}=\partial V / \partial y$, etc. The four parameters for a nondegenerate potential (in addition to the usual additive constant) are the maximum number of parameters that can appear in a superintegrable system. If the number of parameters is fewer than four, we say that the superintegrable potential is degenerate.

The 3D Stäckel transform is a conformal transformation of a superintegrable system on one conformally flat 3D space to a superintegrable system on another such space. We discuss some of the properties of this transform for a classical system and then prove two fundamental results: (1) We show that every superintegrable system with nondegenerate potential is multiseparable. This result uses the structure theory for such systems that we worked out in Ref. 3. (2) We prove that all nondegenerate 3D superintegrable systems are Stäckel transforms of constant curvature systems. Thus, to obtain all nondegenerate conformally flat superintegrable systems, it is sufficient to classify those of constant curvature. The proofs of these fundamental results rest on results obtained in Ref. 3, and the careful reader of this article will need to keep Ref. 3 at hand.

In the second part of the article we use the results of the first part and our explicit knowledge of all separable coordinate systems on 3D constant curvature spaces to make a major advance in the classification of all separable systems with nondegenerate potential on a conformally flat space. Among the separable systems for 3D complex Euclidean space there are seven that are "generic." We give a precise definition later, but, essentially this means that the coordinates belong to a multiparameter family. The ultimate generic coordinates are the Jacobi elliptic coordinates from which all others can be obtained by limiting processes. ${ }^{16,17}$ We show that each of the generic separable systems uniquely determines a nondegenerate superintegrable system that contains it. We obtain a similar result for the five generic separable systems on the complex three-sphere. However, four of these turn out to be Stäckel transforms of Euclidean generic systems. Thus we find eight Stäckel inequivalent generic systems on constant curvature spaces and all generic systems on 3D conformally flat spaces must be Stäckel equivalent to one of these. (In addition there are two nondegenerate superintegrable systems in Euclidean space that are only weakly functionally independent and these give rise to similar systems on a variety of conformally flat spaces.) Thus we exhibit ten families of superintegrable systems in conformally flat spaces. This does not solve the classification problem completely, but it is a major advance. Any remaining nondegenerate superintegrable systems must be multiseparable but separate only in degenerate separable coordinates. This remaining problem is still complicated, but much less so than the original problem. This is a technically detailed proof, but the results are quite explicit and easy to grasp. We derive and give a simple characterization of eight families of separable systems whose Stäckel transforms yield nondegenerate superintegrable systems on a variety of conformally flat spaces.

The next article in this series will extend all of our classical 2D and 3D results to the quantum case. This is very easy in the $2 \mathrm{D}$ case but requires some machinery in $3 \mathrm{D}$. 
Second order conformal Killing tensors: There is a close relationship between the secondorder Killing tensors of a conformally flat space in 3D and the second order conformal Killing tensors of flat space. A second order conformal Killing tensor for a space $M_{3}$ with metric $\mathrm{d} s^{2}$ $=\lambda\left(x_{1}, x_{2}, x_{3}\right)\left(\mathrm{d} x_{1}^{2}+\mathrm{d} x_{2}^{2}+\mathrm{d} x_{3}^{2}\right)$ and free Hamiltonian $\mathcal{H}=\left(p_{1}^{2}+p_{2}^{2}+p_{3}^{2}\right) / \lambda$ is a quadratic form $\mathcal{S}$ $=\sum a^{i j}\left(x_{1}, x_{2}, x_{3}\right) p_{i} p_{j}$ such that $\{\mathcal{H}, \mathcal{S}\}=f\left(x_{1}, x_{2}, x_{3}\right) \mathcal{H}$, for some function $f$. Since $f$ is arbitrary, it is easy to see that $\mathcal{S}$ is a conformal Killing tensor for $M_{3}$ if and only if it is a conformal Killing tensor for flat space $\mathrm{d} x_{1}^{2}+\mathrm{d} x_{2}^{2}+\mathrm{d} x_{3}^{2}$. The conformal Killing tensors for flat space are very well known, e.g., Ref. 18. The space of conformal Killing tensors is infinite dimensional. ${ }^{19}$ It is spanned by products of the conformal Killing vectors

$$
\begin{gathered}
p_{1}, \quad p_{2}, \quad p_{3}, \quad x_{3} p_{2}-x_{2} p_{3}, \quad x_{1} p_{3}-x_{3} p_{1}, \quad x_{2} p_{1}-x_{1} p_{2}, \quad x_{1} p_{1}+x_{2} p_{2}+x_{3} p_{3}, \\
\left(x_{1}^{2}-x_{2}^{2}-x_{3}^{2}\right) p_{1}+2 x_{1} x_{3} p_{3}+2 x_{1} x_{2} p_{2}, \quad\left(x_{2}^{2}-x_{1}^{2}-x_{3}^{2}\right) p_{2}+2 x_{2} x_{3} p_{3}+2 x_{2} x_{1} p_{1}, \\
\left(x_{3}^{2}-x_{1}^{2}-x_{2}^{2}\right) p_{3}+2 x_{3} x_{1} p_{1}+2 x_{3} x_{2} p_{2},
\end{gathered}
$$

and terms $g\left(x_{1}, x_{2}, x_{3}\right)\left(p_{1}^{2}+p_{2}^{2}+p_{3}^{2}\right)$, where $g$ is an arbitrary function. Since every Killing tensor is also a conformal Killing tensor, we see that every second-order Killing tensor for $M_{3}$ can be expressed as a linear combination of these second-order generating elements though, of course, the space of Killing tensors is only finite dimensional. This shows in particular that every $a^{i j}$ and every $a^{i i}-a^{j j}$ with $i \neq j$ is a polynomial of order at most four in $x_{1}, x_{2}, x_{3}$, no matter what is the choice of $\lambda$.

A straightforward, though tedious, computation from the above-mentioned results yields the expressions

$$
\begin{gathered}
a_{11}^{12}=-a_{22}^{12}=\alpha_{3}\left(x_{1}^{2}-x_{2}^{2}\right)+\left(\delta_{1}+\gamma_{2}\right) x_{1} x_{2}-\alpha_{1} x_{1} x_{3}-\alpha_{2} x_{2} x_{3}+\phi_{3} x_{1}+\xi_{3} x_{2}+\mu_{3} x_{3}+\nu_{3}, \\
a_{11}^{13}=-a_{33}^{13}=\alpha_{2}\left(x_{1}^{2}-x_{3}^{2}\right)-\alpha_{1} x_{1} x_{2}+\gamma_{2} x_{1} x_{3}-\alpha_{3} x_{2} x_{3}+\phi_{2} x_{1}+\xi_{2} x_{2}+\mu_{2} x_{3}+\nu_{2}, \\
\alpha_{33}^{23}=-a_{22}^{23}=\alpha_{1}\left(x_{2}^{2}-x_{3}^{2}\right)-\alpha_{2} x_{1} x_{2}+\alpha_{3} x_{1} x_{3}+\delta_{1} x_{2} x_{3}+\phi_{1} x_{1}+\xi_{1} x_{2}+\mu_{1} x_{3}+\nu_{1},
\end{gathered}
$$

where $\alpha_{j}, \delta_{j}, \gamma_{j}, \phi_{j}, \xi_{j}, \mu_{j}$, and $\nu_{j}$ are constants. Further $\left(a^{i i}-a^{j j}\right)_{i}=2 a_{j}^{i j}$ for $i \neq j$, and $a_{3}^{12}+a_{2}^{13}$ $+a_{1}^{23}=0$.

It is useful to pass to new variables $a^{11}, a^{24}, a^{34}, a^{12}, a^{13}, a^{23}$ for the Killing tensor, where $a^{24}=a^{22}-a^{11}, a^{34}=a^{33}-a^{11}$. Then we see that $a^{24}, a^{34}, a^{12}, a^{13}, a^{23}$ are polynomials of order $\leqslant 4$. The remaining conditions can be expressed in the form

$$
\begin{gathered}
\left(a^{11} \lambda\right)_{1}=-\lambda_{2} a^{12}-\lambda_{3} a^{13}, \quad\left(a^{11} \lambda\right)_{2}=-\lambda_{1} a^{12}-\left(a^{24} \lambda\right)_{2}-\lambda_{3} a^{23}, \\
\left(a^{11} \lambda\right)_{3}=-\lambda_{1} a^{13}-\lambda_{2} a^{23}-\left(a^{34} \lambda\right)_{3} .
\end{gathered}
$$

Theorem 1: Necessary and sufficient conditions that the quadratic form $\mathcal{S}=\sum_{i j} a^{i j} p_{i} p_{j}+W$ be a second order constant of the motion for the space with metric $\mathrm{d} s^{2}=\lambda\left(\mathrm{d} x_{1}^{2}+\mathrm{d} x_{2}^{2}+\mathrm{d} x_{3}^{2}\right)$ and potential $V$ are

(1) $\sum_{i j} a^{i j} p_{i} p_{j}$ is a conformal Killing tensor on the flat space with metric $\mathrm{d} x_{1}^{2}+\mathrm{d} x_{2}^{2}+\mathrm{d} x_{3}^{2}$.

(2) The integrability conditions for (3) hold:

$$
\begin{gathered}
\left(\lambda_{2} a^{12}+\lambda_{3} a^{13}\right)_{2}=\left(\lambda_{1} a^{12}+\left(a^{24} \lambda\right)_{2}+\lambda_{3} a^{23}\right)_{1}, \\
\left(\lambda_{2} a^{12}+\lambda_{3} a^{13}\right)_{3}=\left(\lambda_{1} a^{13}+\lambda_{2} a^{23}+\left(a^{34} \lambda\right)_{3}\right)_{1}, \\
\left(\lambda_{1} a^{12}+\left(a^{24} \lambda\right)_{2}+\lambda_{3} a^{23}\right)_{3}=\left(\lambda_{1} a^{13}+\lambda_{2} a^{23}+\left(a^{34} \lambda\right)_{3}\right)_{2} .
\end{gathered}
$$

The Bertrand-Darboux conditions for the potential hold: 


$$
\sum_{s=1}^{3}\left[V_{s j} \lambda a^{s \ell}-V_{s \ell} \lambda a^{s j}+V_{s}\left(\left(\lambda a^{s \ell}\right)_{j}-\left(\lambda a^{s j}\right)_{\ell}\right)\right]=0 .
$$

These are just the conditions $\partial_{x_{\ell}} W_{j}=\partial_{x_{j}} W_{\ell}$ for $j \neq \ell$.

\section{THE STÄCKEL TRANSFORM FOR 3D SYSTEMS}

The Stäckel transform ${ }^{14}$ or coupling constant metamorphosis ${ }^{15}$ plays a fundamental role in relating superintegrable systems on different manifolds. Suppose we have a superintegrable system

$$
H=\frac{p_{1}^{2}+p_{2}^{2}+p_{3}^{2}}{\lambda(x, y, z)}+V(x, y, z)
$$

in local orthogonal coordinates, with nondegenerate potential $V(x, y, z)$ :

$$
\begin{gathered}
V_{33}=V_{11}+A^{33} V_{1}+B^{33} V_{2}+C^{33} V_{3}, \quad V_{22}=V_{11}+A^{22} V_{1}+B^{22} V_{2}+C^{22} V_{3}, \\
V_{23}=A^{23} V_{1}+B^{23} V_{2}+C^{23} V_{3}, \quad V_{13}=A^{13} V_{1}+B^{13} V_{2}+C^{13} V_{3}, \\
V_{12}=A^{12} V_{1}+B^{12} V_{2}+C^{12} V_{3}
\end{gathered}
$$

and suppose $U(x, y, z)$ is a particular solution of Eqs. (7), nonzero in an open set. Then the transformed system $\widetilde{H}=\left(p_{1}^{2}+p_{2}^{2}+p_{3}^{2}\right) / \widetilde{\lambda}+\widetilde{V}$ with nondegenerate potential $\widetilde{V}(x, y, z)$ :

$$
\begin{aligned}
& \tilde{V}_{33}=\widetilde{V}_{11}+\widetilde{A}^{33} \widetilde{V}_{1}+\widetilde{B}^{33} \widetilde{V}_{2}+\widetilde{C}^{33} \widetilde{V}_{3}, \quad \widetilde{V}_{22}=\widetilde{V}_{11}+\widetilde{A}^{22} \widetilde{V}_{1}+\widetilde{B}^{22} \widetilde{V}_{2}+\widetilde{C}^{22} \widetilde{V}_{3}, \\
& \widetilde{V}_{23}=\widetilde{A}^{23} \widetilde{V}_{1}+\widetilde{B}^{23} \widetilde{V}_{2}+\widetilde{C}^{23} \widetilde{V}_{3}, \quad \widetilde{V}_{13}=\widetilde{A}^{13} \widetilde{V}_{1}+\widetilde{B}^{13} \widetilde{V}_{2}+\widetilde{C}^{13} \widetilde{V}_{3}, \\
& \widetilde{V}_{12}=\widetilde{A}^{12} \widetilde{V}_{1}+\widetilde{B}^{12} \widetilde{V}_{2}+\widetilde{C}^{12} \widetilde{V}_{3},
\end{aligned}
$$

is also superintegrable, where

$$
\begin{gathered}
\tilde{\lambda}=\lambda U, \quad \tilde{V}=\frac{V}{U}, \quad \widetilde{A}^{33}=A^{33}+2 \frac{U_{1}}{U}, \quad \widetilde{C}^{33}=C^{33}-2 \frac{U_{3}}{U}, \\
\widetilde{A}^{22}=A^{22}+2 \frac{U_{1}}{U}, \quad \widetilde{B}^{22}=B^{22}-2 \frac{U_{2}}{U}, \quad \widetilde{B}^{23}=B^{23}-\frac{U_{3}}{U}, \quad \widetilde{C}^{23}=C^{23}-\frac{U_{2}}{U}, \\
\widetilde{A}^{13}=A^{13}-\frac{U_{3}}{U}, \quad \widetilde{C}^{13}=C^{13}-\frac{U_{1}}{U}, \quad \widetilde{A}^{12}=A^{12}-\frac{U_{2}}{U}, \quad \widetilde{B}^{12}=B^{12}-\frac{U_{1}}{U},
\end{gathered}
$$

and $\widetilde{A}^{23}=A^{23}, \widetilde{B}^{33}=B^{33}, \widetilde{B}^{13}=B^{13}, \widetilde{C}^{22}=C^{22} 4, \widetilde{C}^{12}=C^{12}$. Let $S=\sum a^{i j} p_{i} p_{j}+W=S_{0}+W$ be a second order symmetry of $H$ and $S_{U}=\sum a^{i j} p_{i} p_{j}+W_{U}=S_{0}+W_{U}$ be the special case that is in involution with $\left(p_{1}^{2}+p_{2}^{2}+p_{3}^{2}\right) / \lambda+U$. Then $\tilde{S}=S_{0}-\left(W_{U} / U\right) H+(1 / U) H$ is the corresponding symmetry of $\tilde{H}$. Since one can always add a constant to a nondegenerate potential, it follows that $1 / U$ defines an inverse Stäckel transform of $\widetilde{H}$ to $H$. See Ref. 14 for many examples of this transform.

\section{MULTISEPARABILITY AND STÄCKEL EQUIVALENCE}

From the general theory of variable separation for Hamilton-Jacobi equations, e.g., Refs. 20 and 21 we know that second order symmetries $L_{1}, L_{2}$ define a separable system for the equation 


$$
H=\frac{p_{x}^{2}+p_{y}^{2}+p_{z}^{2}}{\lambda(x, y, z)}+V(x, y, z)=E
$$

if and only if (1) the symmetries $H, L_{1}, L_{2}$ form a linearly independent set as quadratic forms, (2) $\left\{L_{1}, L_{2}\right\}=0$, and, (3) the three quadratic forms have a common eigenbasis of differential forms. This last requirement means that, expressed in coordinates $x, y$, and $z$, at least one of the matrices $\mathcal{A}_{(j)}(\mathbf{x})$ (of the quadratic form associated with $L_{j}$ ) can be diagonalized by conjugacy transforms in a neighborhood of a regular point and that $\left[\mathcal{A}_{(2)}(\mathbf{x}), \mathcal{A}_{(1)}(\mathbf{x})\right]=0$. However, for nondegenerate superintegrable potentials in a conformally flat space we see that $\left\{L_{1}, L_{2}\right\}=0 \leftrightarrow\left[\mathcal{A}_{(2)}\left(\mathbf{x}_{0}\right), \mathcal{A}_{(1)}\right.$ $\left.\times\left(\mathbf{x}_{0}\right)\right]=0, \mathcal{F}\left(\mathbf{x}_{0}\right)=0$ at a single regular point $\mathbf{x}_{0}$, see Sec. V of Ref. 3, so that the intrinsic conditions for the existence of a separable coordinate system are simplified.

Let $\mathcal{A}=\sum_{i \leqslant j} a^{i j} \mathcal{A}^{i j}, \mathcal{B}=\sum_{i \leqslant j} b^{i j} \mathcal{A}^{i j}$, be the matrices of two symmetries at the point $\mathbf{x}_{0}$. Here, $\mathcal{A}^{i j}=\frac{1}{2}\left(\mathcal{E}^{i j}+\mathcal{E}^{j i}\right)$ where $\mathcal{E}^{i j}$ is the $3 \times 3$ matrix with matrix element 1 in row $i$, column $j$, and 0 everywhere else. From the table in Sec. V of Ref. 3 we see that the corresponding symmetries are in involution if and only if the matrices $\mathcal{A}, \mathcal{B}$ commute and the additional condition

$$
\begin{aligned}
& \left(a^{12} b^{11}-b^{12} a^{11}\right)\left(C^{33}-B^{23}-A^{13}\right)+\left(a^{22} b^{12}-a^{12} b^{22}\right)\left(C^{33}-2 B^{23}\right) \\
& \quad+\left(a^{13} b^{11}-a^{11} b^{13}\right)\left(B^{33}+2 A^{12}-B^{22}\right)+\left(a^{33} b^{13}-a^{13} b^{33}\right)\left(2 B^{33}+2 A^{12}-B^{22}\right) \\
& +\left(a^{23} b^{22}-a^{22} b^{23}\right)\left(-2 B^{12}-A^{33}\right)+\left(a^{33} b^{23}-b^{33} a^{23}\right)\left(-2 B^{12}+A^{22}-2 A^{33}\right) \\
& +2\left(a^{11} b^{22}-a^{22} b^{11}+a^{33} b^{11}-a^{11} b^{33}+a^{22} b^{33}-a^{33} b^{22}\right) A^{23}+\left(a^{23} b^{11}-a^{11} b^{23}\right)\left(A^{22}-A^{33}\right) \\
& +\left(a^{33} b^{12}-a^{12} b^{33}\right)\left(B^{23}-A^{13}\right)+\left(a^{13} b^{22}-a^{22} b^{13}\right) B^{33}=0
\end{aligned}
$$

holds. Note that the metric $G$ does not appear in these conditions.

Theorem 2: Let $V$ be a superintegrable nondegenerate potential in a $3 D$ conformally flat space. Then $V$ defines a multiseparable system.

Proof: From (9) we see that the second order symmetries with matrices $\mathcal{A}^{(33)}$ and $\alpha \mathcal{A}^{(11)}$ $+\beta \mathcal{A}^{(12)}$ will be in involution if and only if $2 \alpha A^{23}+\beta\left(B^{23}-A^{13}\right)=0$ at the regular point $\mathbf{x}_{0}$. If $A^{23}\left(\mathbf{x}_{0}\right)=0$ we can set $\alpha=1, \beta=0$ and the symmetries $\mathcal{A}^{(33)}, \mathcal{A}^{11}$ will define a separable system. If $A^{23}\left(\mathbf{x}_{0}\right) \neq 0$ we can set $\alpha=-\left(B^{23}-A^{13}\right) / 2 A^{23}, \beta=1$. Then the symmetries with nonzero matrices $\mathcal{A}^{(33)}$ and $\alpha \mathcal{A}^{(11)}+\beta \mathcal{A}^{(12)}$ will be in involution. The second case must occur for some regular point $\mathbf{x}_{0}$ unless $A^{23}(\mathbf{x})=0$ for all $\mathbf{x}$. In this last eventuality we can perform a suitable Euclidean rotation (with arbitrarily small complex rotation angle) so that $A^{23}$ does not vanish identically in the rotated coordinate system. It is a straightforward exercise to show that this transformation is not possible if and only if

$$
B^{33}=C^{22}=0, \quad A^{13}=B^{23}, \quad A^{12}=C^{23}, \quad A^{22}=A^{33} .
$$

In this eventuality, we can set $\alpha=0, \beta=1$ and find a solution. Thus we can always find a linear combination of these matrices, corresponding to $\beta=1$ and with three distinct eigenvalues, so they will determine separable coordinates. We could have carried through this same construction for the second order symmetries with matrices $\mathcal{A}^{(22)}$ and $\gamma \mathcal{A}^{(11)}+\delta \mathcal{A}^{(13)}$ and for the second order symmetries with matrices $\mathcal{A}^{(11)}$ and $\mu \mathcal{A}^{(22)}+\xi \mathcal{A}^{(23)}$ and shown that we could always find solutions with $\delta=\xi=1$. Thus the system is multiseparable (in at least three coordinate systems). Q.E.D.

Corollary 1: Let $V$ be a superintegrable nondegenerate potential in a $3 D$ conformally flat space. Then there is a continuous one-parameter (or multiparameter) family of separable systems for $V$, spanning at least a five-dimensional subspace of symmetries.

Proof: We follow the method of proof of the theorem.

Case I: Suppose $A^{23}\left(\mathbf{x}_{0}\right) \neq 0$. From (9) we can verify that the symmetries with matrices 


$$
\mathcal{A}=\left(\begin{array}{ccc}
0 & 0 & 0 \\
0 & f^{2} & -f \\
0 & -f & 1
\end{array}\right), \quad \mathcal{B}=\left(\begin{array}{ccc}
g & 1 / 2 & f / 2 \\
1 / 2 & 0 & 0 \\
f / 2 & 0 & 0
\end{array}\right),
$$

are in involution provided

$$
\begin{gathered}
-4 f^{2} B^{23}+2 f^{2} C^{33}+3 f B^{33}+4 f A^{12}-2 f B^{22}+f B^{23}-4 f^{2} g A^{23}-4 f g A^{22} \\
+4 f g A^{33}+4 g A^{23}+2 B^{23}-2 A^{13}-2 f^{3} B^{33}=0 .
\end{gathered}
$$

As $A^{23}\left(\mathbf{x}_{0}\right) \neq 0$ this equation can be solved for $g$ as a function of $f$ for $f$ in some open set. The resulting symmetries $\mathcal{A}, \mathcal{B}$ are in involution and have eigenvalues $\left(0,0, f^{2}+1\right)$ and $\left(0, \frac{1}{2}[g\right.$ $\left.+\sqrt{f^{2}+g^{2}+1}\right], \frac{1}{2}\left[g-\sqrt{f^{2}+g^{2}+1}\right]$, respectively. Thus they determine a one-parameter family of separable coordinates. Moreover, as $f$ varies in an open set, the space spanned by the symmetries (including the Hamiltonian) has dimension six.

Case II: If $A^{23}\left(\mathbf{x}_{0}\right)=0$, we can assume that Eq. (10) holds. Then the problem breaks up into a series of special cases. Suppose first that $C^{33}-2 A^{13}=\ell \neq 0$. Then we can verify that the symmetries with matrices

$$
\mathcal{A}=\left(\begin{array}{lll}
0 & 2 g k / K & 0 \\
2 g k / K & 1 & -g / K \\
0 & -g / K & g^{2} / K
\end{array}\right), \quad \mathcal{B}=\left(\begin{array}{lll}
f & g / 2 & 1 / 2 \\
g / 2 & 0 & 0 \\
1 / 2 & 0 & k
\end{array}\right),
$$

are in involution provided $K=1-4 f k \neq 0$ and $g$ satisfies

$$
-g\left(2 A^{12}-B^{22}\right)+2 k\left(A^{22}+2 B^{12}\right)=\ell .
$$

If $2 A^{12}-B^{22} \neq 0$ then there is a nonzero solution expression $g$ as a function of $k$. Since $f, k$ are essentially arbitrary, they determine a five-dimensional space spanned by the symmetries and a two-parameter family of separable coordinates. If $2 A^{12}-B^{22}=0, A^{22}+2 B^{12} \neq 0$ then $k$ is a nonzero constant and $f, g$ are essentially arbitrary, so they again determine a five-dimensional space spanned by the symmetries and a two-parameter family of separable coordinates. If $2 A^{12}-B^{22}$ $=0, A^{22}+2 B^{12}=0$, then the symmetries with matrices

$$
\mathcal{A}=\left(\begin{array}{lll}
0 & H / K & 0 \\
H / K & 1 & 0 \\
0 & 0 & L / K
\end{array}\right), \quad \mathcal{B}=\left(\begin{array}{lll}
f & g / 2 & 1 / 2 \\
g / 2 & 0 & h / 2 \\
1 / 2 & h / 2 & k
\end{array}\right),
$$

where $K=1-4 f k-h^{2}-2 h g f \neq 0$, and

$$
H=-h+2 g k+h g^{2}, \quad G=-g+2 f h+g h^{2}, \quad L=g^{2}-h^{2}+2 h g(k-f),
$$

are in involution provided $f=(g / 2 h-h / 2 g$ ). (This implies $G=0$ and $L=H h$.) They determine a six-dimensional space spanned by the symmetries and a three-parameter family of separable coordinates. This covers all cases where $\ell \neq 0$.

Now suppose $\ell=0$, i.e., $C^{33}=2 A^{13}$. Then the symmetries with matrices (13) are in involution provided

$$
\left(2 A^{12}-B^{22}\right)\left(h^{2}-g^{2}+2 h g f-2 h k g\right)+\left(A^{22}+2 B^{12}\right)\left(-h+2 k g+h g^{2}\right)=0 .
$$

If $2 A^{12}-B^{22} \neq 0$ then we can solve this equation to express $f$ as a nonzero function of $g, h, k$. This yields at least a five-dimensional space spanned by the symmetries and a three-parameter family of separable coordinates. Finally, suppose in addition that $2 A^{12}-B^{22}=0$. Then we can verify that the symmetries with matrices 


$$
\mathcal{A}=\left(\begin{array}{lll}
0 & 0 & 0 \\
0 & f^{2} & -f \\
0 & -f & 1
\end{array}\right), \quad \mathcal{B}=\left(\begin{array}{lll}
g & 1 / 2 & f / 2 \\
1 / 2 & 0 & 0 \\
f / 2 & 0 & 0
\end{array}\right),
$$

are in involution with no conditions on $f, g$. Again, as $f, g$ vary in an open set, the space spanned by the symmetries (including the Hamiltonian) has dimension six.

In Ref. 17 the following result was obtained.

Theorem 3: Let $u_{1}, u_{2}, u_{3}$ be an orthogonal separable coordinate system for a $3 D$ conformally flat space with metric $\mathrm{d} \widetilde{s}^{2}$. Then there is a function $f$ such that $f \mathrm{~d} \widetilde{s}^{2}=\mathrm{d} s^{2}$ where $\mathrm{d} s^{2}$ is a constant curvature space metric and $\mathrm{d} s^{2}$ is orthogonally separable in exactly these same coordinates $u_{1}, u_{2}, u_{3}$. The function $f$ is called a Stäckel multiplier with respect to this coordinate system.

Thus the possible separable coordinate systems for a conformally flat space are all obtained, modulo a Stäckel multiplier, from separable systems on 3D flat space or on the three sphere.

Theorem 4: Every superintegrable system with nondegenerate potential on a 3D conformally flat space is Stäckel equivalent to a superintegrable system on either $3 D$ flat space or the three sphere.

Proof: Suppose we have a superintegrable system with nondegenerate potential on a conformally flat space. Then by Corollary 1 this system separates in a one- or multiparameter family of coordinate systems spanning a five-dimensional subspace of symmetries. By Theorem 3 each of these three systems is conformal to a separable system in flat space or on the three sphere. Thus from Ref. 19, p. 85, the metric for the space in standard Cartesian-like coordinates $x, y, z$ is simultaneously conformal to three systems corresponding to the following possible choices for the metric function $\lambda(x, y, z)$, namely

$$
1, \quad 1 /(x+i y)^{2}, \quad 1 / r^{4} \quad \text { (flatspace); } 1 / x^{2}, \quad 1 /\left(1+r^{2} / 4\right)^{2} \quad \text { (three sphere); }
$$

in the same coordinates, and each of the conformal factors is a Stäckel multiplier with respect to the corresponding separable coordinates. From the Corollary we see that we can find two separable systems such that the factor (15) is the same, i.e., the metric must take the form $\mathrm{d} \widetilde{s}^{2}=f \mathrm{~d} s^{2}$, where $\mathrm{d} s^{2}$ is the metric on a single constant curvature space, either 3D flat space or the three sphere, and the constant curvature space separates in these same two coordinate systems. Further the space of symmetries spanned by the two sets is at least five dimensional.

Then we have $(\tilde{\mathcal{H}}+\widetilde{V}) / f=\mathcal{H}+V$, where $\widetilde{\mathcal{H}}+\widetilde{V}$ is the original superintegrable system, $\mathcal{H}$ is the Hamiltonian on a constant curvature space, and $V$ is the induced multiparameter potential. Under the transform $f$ each of the commuting second order symmetries $\mathcal{S}$ of the original system that defines a coordinate separation transforms to a symmetry of the form $\mathcal{S}+g_{S} \mathcal{H}$ for $g_{S}$ a function. There are at least five such functionally linearly independent symmetries arising from separation in two coordinate systems, so the constant curvature space system admits five functionally linearly independent symmetries. Thus the potential $V$ must satisfy the Bertrand-Darboux equations for these symmetries. It follows that $V$ is nondegenerate and by Theorem 2 of Ref. 3 that the system $\mathcal{H}+V$ is itself superintegrable with nondegenerate potential. The function $f$ is simultaneously a Stäckel multiplier with respect to the two coordinate systems whose symmetries completely characterize the superintegrable system $\mathcal{H}+V$. That is, $f$ satisfies the Bertrand-Darboux equations for five functionally linearly independent symmetries. Hence $f$ itself satisfies the equations that determine the nondegenerate potential $V$. This means that the system $\widetilde{\mathcal{H}}+\widetilde{V}$ is Stäckel equivalent to the constant curvature space superintegrable system.

Q.E.D.

\section{CLASSIFICATION OF NONDEGENERATE SYSTEMS}

\section{A. Separable systems in complex Euclidean space}

It is a difficult task to list all 3D conformally flat superintegrable systems with nondegenerate potential and to show that the classification is complete. However, we now have tools to simplify the problem. First, as every such system is Stäckel equivalent to a system on Euclidean space or the complex sphere, we can restrict ourselves to those two spaces. Second, since every such 
system is multiseparable, we can bring to bear our knowledge of all orthogonal separable coordinates on these spaces. These results can be gleaned from the books ${ }^{20,16}$ and many papers of the authors, e.g., Ref. 17. Thus in principle, we have enough information to accomplish our task, though the details are formidably complicated.

We begin by summarizing the full list of orthogonal separable systems in complex Euclidean space and the associated symmetry operators. Here, a "natural" basis for first order symmetries is given by $p_{1} \equiv p_{x}, p_{2} \equiv p_{y}, p_{3} \equiv p_{z}, J_{1}=y p_{z}-z p_{y}, J_{2}=z p_{x}-x p_{z}, J_{3}=x p_{y}-y p_{x}$ in the classical case and $p_{1}=\partial_{x}, p_{2}=\partial_{y}, p_{3}=\partial_{z}, J_{1}=y \partial_{z}-z \partial_{y}, J_{2}=z \partial_{x}-x \partial_{z}, J_{3}=x \partial_{y}-y \partial_{x}$ in the quantum case. (In the operator characterizations for the quantum case, the classical product of two constants of the motion is replaced by the symmetrized product of the corresponding operator symmetries.) The Hamiltonian is $H=p_{1}^{2}+p_{2}^{2}+p_{3}^{2}$. In each case below we list the coordinates followed by the constants of the motion that characterize them.

Note: The bracket notation used to describe generic coordinates in three-dimensional Euclidean space is due to Bôcher and is an adaptation of the notation used to describe the elementary divisors of two quadratic forms one of which is the quadratic form associated with Euclidean space and the second with the quadratic form of the coordinate curves describing the coordinate system. In order to do this in three dimensions and also deal with separable solutions of Laplace's equation we use the symbol $\left[p_{0}, p_{1}, \ldots, p_{r}\right]$ where $\sum_{i=0}^{r} p_{i}=5$ and $p_{0} \geqslant 2$. (See Ref. 16 for further details). This determines a coordinate system whose infinitesimal distance is of the form

$$
\mathrm{d} s^{2}=\frac{1}{(u-v)(u-w)} P(u) p_{u}^{2}+\frac{1}{(v-u)(v-w)} P(v) p_{v}^{2}+\frac{1}{(w-v)(w-u)} P(w) p_{w}^{2},
$$

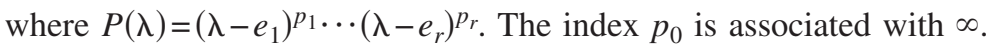

$$
\begin{aligned}
& \text { [2111] } \\
& x^{2}=c^{2} \frac{\left(u-e_{1}\right)\left(v-e_{1}\right)\left(w-e_{1}\right)}{\left(e_{1}-e_{2}\right)\left(e_{1}-e_{3}\right)}, \quad y^{2}=c^{2} \frac{\left(u-e_{2}\right)\left(v-e_{2}\right)\left(w-e_{2}\right)}{\left(e_{2}-e_{1}\right)\left(e_{2}-e_{3}\right)}, \\
& z^{2}=c^{2} \frac{\left(u-e_{3}\right)\left(v-e_{3}\right)\left(w-e_{3}\right)}{\left(e_{3}-e_{1}\right)\left(e_{3}-e_{2}\right)}, \\
& L_{1}=J_{1}^{2}+J_{2}^{2}+J_{3}^{2}+c^{2}\left(\left(e_{1}+e_{2}\right) p_{3}^{2}+\left(e_{1}+e_{3}\right) p_{2}^{2}+\left(e_{3}+e_{2}\right) p_{1}^{2}\right), \\
& L_{2}=e_{1} J_{1}^{2}+e_{2} J_{2}^{2}+e_{3} J_{3}^{2}+c^{2}\left(e_{1} e_{2} p_{3}^{2}+e_{1} e_{3} p_{2}^{2}+e_{3} e_{2} p_{1}^{2}\right) \text {. } \\
& \text { [211] } x^{2}+y^{2}=-c^{2}\left[\frac{\left(u-e_{1}\right)\left(v-e_{1}\right)\left(w-e_{1}\right)}{\left(e_{1}-e_{2}\right)^{2}}\right] \\
& -\frac{c^{2}}{e_{1}-e_{2}}\left[\left(u-e_{1}\right)\left(v-e_{1}\right)+\left(u-e_{1}\right)\left(w-e_{1}\right)+\left(v-e_{1}\right)\left(w-e_{1}\right)\right] \text {, } \\
& (x-i y)^{2}=c^{2} \frac{\left(u-e_{1}\right)\left(v-e_{1}\right)\left(w-e_{1}\right)}{e_{1}-e_{2}}, \quad z^{2}=c^{2} \frac{\left(u-e_{2}\right)\left(v-e_{2}\right)\left(w-e_{2}\right)}{\left(e_{2}-e_{1}\right)^{2}} . \\
& L_{1}=J_{1}^{2}+J_{2}^{2}+J_{3}^{2}+c^{2}\left(\left(e_{1}-e_{2}\right)\left(p_{1}+i p_{2}\right)^{2}+2 e_{2} p_{3}^{2}+\left(e_{1}+e_{2}\right)\left(p_{1}^{2}+p_{2}^{2}\right)\right), \\
& L_{2}=e_{2}\left(J_{1}^{2}+J_{2}^{2}\right)+\left(e_{2}-e_{1}\right)\left(J_{1}+i J_{2}\right)^{2}+e_{1} J_{3}^{2}+c^{2}\left(\left(e_{1} e_{2}\left(p_{1}^{2}+p_{2}^{2}\right)+e_{1}\left(e_{1}-e_{2}\right)\left(p_{1}+i p_{2}\right)^{2}+e_{2}^{2} p_{3}^{2}\right)\right) . \\
& \text { [23] } x-i y=\frac{1}{2} c\left(\frac{u^{2}+v^{2}+w^{2}}{u v w}-\frac{1}{2} \frac{u^{2} v^{2}+u^{2} w^{2}+v^{2} w^{2}}{u^{3} v^{3} w^{3}}\right) \text {, }
\end{aligned}
$$




$$
\begin{gathered}
z=\frac{1}{2} c\left(\frac{u v}{w}+\frac{u w}{v}+\frac{v w}{u}\right), \quad x+i y=c u v w . \\
L_{1}=J_{1}^{2}+J_{2}^{2}+J_{3}^{2}+2 c^{2}\left(p_{1}+i p_{2}\right) p_{3}, \quad L_{2}=-2 J_{3}\left(J_{1}+i J_{2}\right)+c^{2}\left(p_{1}+i p_{2}\right)^{2} . \\
{[311] \quad x=\frac{c}{4}\left(u^{2}+v^{2}+w^{2}+\frac{1}{u^{2}}+\frac{1}{v^{2}}+\frac{1}{w^{2}}\right)+\frac{3}{2} c,} \\
y=-\frac{c}{4} \frac{\left(u^{2}-1\right)\left(v^{2}-1\right)\left(w^{2}-1\right)}{u v w}, \quad z=i \frac{c}{4} \frac{\left(u^{2}+1\right)\left(v^{2}+1\right)\left(w^{2}+1\right)}{u v w} . \\
L_{1}=c\left(J_{3} p_{2}-J_{2} p_{3}\right)+c^{2}\left(p_{1}^{2}-p_{2}^{2}\right), \quad L_{2}=-\frac{1}{4} J_{1}^{2}-c J_{2} p_{3}-c^{2} p_{3}^{2} . \\
{[32] \quad x+i y=u v w, \quad x-i y=-\left(\frac{u v}{w}+\frac{u w}{v}+\frac{v w}{u}\right), \quad z=\frac{1}{2}\left(u^{2}+v^{2}+w^{2}\right) .} \\
L_{1}=-c\left(J_{2}+i J_{1}\right)\left(p_{1}+i p_{2}\right)-c\left(J_{2}-i J_{1}\right)\left(p_{1}-i p_{2}\right)-c^{2}\left(p_{1}+i p_{2}\right)^{2}, \\
L_{2}=J_{3}^{2}-2 c\left(J_{2}-i J_{1}\right)\left(p_{1}+i p_{2}\right) .
\end{gathered}
$$

[41] $x+i y=u^{2} v^{2}+u^{2} w^{2}+v^{2} w^{2}-\frac{1}{2}\left(u^{4}+v^{4}+w^{4}\right), \quad x-i y=c^{2}\left(u^{2}+v^{2}+w^{2}\right), \quad z=2 i c u v w$.

The symmetries that describe this system are

$$
\begin{gathered}
L_{1}=-i J_{3}\left(p_{1}-i p_{2}\right)+\left(J_{2}+i J_{1}\right) p_{3}+\frac{1}{4} c^{4}\left(p_{1}+i p_{2}\right)^{2}, \quad L_{2}=-\left(J_{1}-i J_{2}\right)^{2}-2 i c^{4}\left(J_{1}+i J_{2}\right) p_{3} . \\
{[5] \quad x+i y=c(u+v+w), \quad x-i y=\frac{c}{4}(u-v-w)(u+v-w)(u+w-v),} \\
z=-\frac{c}{4}\left(u^{2}+v^{2}+w^{2}-2(u v+u w+v w)\right) . \\
L_{1}=i J_{3}\left(p_{1}+i p_{2}\right)+\left(J_{2}-i J_{1}\right) p_{3}+c p_{3}\left(p_{1}-i p_{2}\right), \\
L_{2}=\frac{1}{4}\left(J_{2}-i J_{1}\right)^{2}-c\left(2\left(J_{2}+i J_{1} i\left(p_{1}+i p_{2}\right)+i\left(p_{1}-i p_{2}\right)\left(J_{1}+i J_{2}\right)\right)+\frac{c^{2}}{4}\left(p_{1}-i p_{2}\right)^{2} .\right.
\end{gathered}
$$

We summarize the remaining degenerate separable coordinates:

Euclidean coordinates: All of these have one symmetry in common: $L_{1}=p_{3}^{2}$. The seven systems are, polar, Cartesian, light cone, elliptic, parabolic, hyperbolic, and semihyperbolic.

Complex sphere coordinates: These all have the symmetry $L_{1}=J_{1}^{2}+J_{2}^{2}+J_{3}^{2}$ in common. The five systems are spherical, horospherical, elliptical, hyperbolic, and semicircular parabolic.

Rotational types of coordinates: There are three of these systems, each of which is characterized by the fact that one defining symmetry is a perfect square.

Nonorthogonal heat type coordinates: Each of these nonorthogonal systems corresponds to one first order symmetry. Hence it cannot arise for systems with nondegenerate potentials. 
Note that the first seven separable systems are generic, i.e., they occur in one-, two- or three-parameter families, whereas the remaining systems are special limiting cases of the generic ones. We shall show that each of the generic separable systems uniquely determines a nondegenerate superintegrable system.

\section{B. Generic 3D Euclidean superintegrable systems}

Each of the seven generic Euclidean separable systems depends on a scaling parameter $c$ and up to three parameters $e_{1}, e_{2}, e_{3}$. For each such set of coordinates we shall show that there is exactly one nondegenerate superintegrable system that admits separation in these coordinates simultaneously for all values of the parameters $c, e_{j}$.

Consider the system in Ref. 23, for example. If a nondegenerate superintegrable system separates in these coordinates for all values of the parameter $c$, then the space of second order symmetries must contain the five symmetries

$$
\begin{gathered}
\mathcal{H}=p_{x}^{2}+p_{y}^{2}+p_{z}^{2}+V, \quad \mathcal{S}_{1}=J_{1}^{2}+J_{2}^{2}+J_{3}^{2}+f_{1}, \quad \mathcal{S}_{2}=J_{3}\left(J_{1}+i J_{2}\right)+f_{2}, \\
\mathcal{S}_{3}=\left(p_{x}+i p_{y}\right)^{2}+f_{3}, \quad \mathcal{S}_{4}=p_{z}\left(p_{x}+i p_{y}\right)+f_{4} .
\end{gathered}
$$

It is straightforward to check that the $12 \times 5$ matrix of coefficients of the second derivative terms in the twelve Bertrand-Darboux equations associated with symmetries $\mathcal{S}_{1}, \ldots, \mathcal{S}_{4}$ has rank five in general. Thus, there is at most one nondegenerate superintegrable system admitting these symmetries. Solving the Bertrand-Darboux equations for the potential we find the unique solution

$$
V(\mathbf{x}):=\alpha\left(x^{2}+y^{2}+z^{2}\right)+\frac{\beta}{(x+i y)^{2}}+\frac{\gamma z}{(x+i y)^{3}}+\frac{\delta\left(x^{2}+y^{2}-3 z^{2}\right)}{(x+i y)^{4}} .
$$

Finally, we can use the symmetry conditions for this potential to obtain the full six-dimensional space of second order symmetries. This is the superintegrable system III on the following table. The other six cases yield corresponding results.

Theorem 5: Each of the seven generic Euclidean separable systems determines a unique nondegenerate superintegrable system that permits separation simultaneously for all values of the scaling parameter $c$ and any other defining parameters $e_{j}$. For each of these systems there is a basis of five (strongly) functionally independent and six linearly independent second order symmetries. The corresponding nondegenerate potentials and basis of symmetries are (the $f_{j}$ are functions of $\left.x_{1}, x_{2}, x_{3}\right)$ :

$$
\begin{gathered}
\text { I }[2111] \quad V=\frac{\alpha_{1}}{x^{2}}+\frac{\alpha_{2}}{y^{2}}+\frac{\alpha_{3}}{z^{2}}+\delta\left(x^{2}+y^{2}+z^{2}\right), \\
\mathcal{P}_{i}=\partial_{x_{i}}^{2}+\delta x_{i}^{2}+\frac{\alpha_{i}}{x_{i}^{2}}, \quad \mathcal{J}_{i j}=\left(x_{i} p_{x_{j}}-x_{j} p_{x_{i}}\right)^{2}+\alpha_{i}^{2} \frac{x_{j}^{2}}{x_{i}^{2}}+\alpha_{j}^{2} \frac{x_{i}^{2}}{x_{j}^{2}}, \quad i \geqslant j . \\
\mathrm{II}[221] \quad V=\alpha\left(x^{2}+y^{2}+z^{2}\right)+\beta \frac{x-i y}{(x+i y)^{3}}+\frac{\gamma}{(x+i y)^{2}}+\frac{\delta}{z^{2}}, \\
\mathcal{S}_{1}=J \cdot J+f_{1}, \quad \mathcal{S}_{2}=p_{z}^{2}+f_{2}, \quad \mathcal{S}_{3}=J_{3}^{2}+f_{3}, \\
\mathcal{S}_{4}=\left(p_{x}+i p_{y}\right)^{2}+f_{4}, \quad L_{5}=\left(J_{2}-i J_{1}\right)^{2}+f_{5} .
\end{gathered}
$$




$$
\begin{gathered}
\text { III [23] } V=\alpha\left(x^{2}+y^{2}+z^{2}\right)+\frac{\beta}{(x+i y)^{2}}+\frac{\gamma z}{(x+i y)^{3}}+\frac{\delta\left(x^{2}+y^{2}-3 z^{2}\right)}{(x+i y)^{4}}, \\
\mathcal{S}_{1}=J \cdot J+f_{1}, \quad \mathcal{S}_{2}=\left(J_{2}-i J_{1}\right)^{2}+f_{2}, \quad \mathcal{S}_{3}=J_{3}\left(J_{2}-i J_{1}\right)+f_{3}, \\
\mathcal{S}_{4}=\left(p_{x}+i p_{y}\right)^{2}+f_{4}, \quad \mathcal{S}_{5}=p_{z}\left(p_{x}+i p_{y}\right)+f_{5} . \\
\mathrm{IV}[311] \quad V=\alpha\left(4 x^{2}+y^{2}+z^{2}\right)+\beta x+\frac{\gamma}{y^{2}}+\frac{\delta}{z^{2}}, \\
\mathcal{S}_{1}=p_{x}^{2}+f_{1}, \quad \mathcal{S}_{2}=p_{y}^{2}+f_{2}, \quad \mathcal{S}_{3}=p_{z} J_{2}+f_{3}, \\
\mathcal{S}_{4}=p_{y} J_{3}+f_{4}, \quad \mathcal{S}_{5}=J_{1}^{2}+f_{5} . \\
\mathrm{V}[32] \quad V=\alpha\left(4 x^{2}+y^{2}+z^{2}\right)+\beta x+\frac{\gamma}{(y+i z)^{2}}+\frac{\delta(y-i z)}{(y+i z)^{3}}, \\
\mathcal{S}_{1}=p_{x}^{2}+f_{1}, \quad \mathcal{S}_{2}=J_{1}^{2}+f_{2}, \quad \mathcal{S}_{3}=\left(p_{z}-i p_{y}\right)\left(J_{2}+i J_{3}\right)+f_{3}, \\
\mathcal{S}_{4}=p_{z} J_{2}-p_{y} J_{3}+f_{4}, \quad \mathcal{S}_{5}=\left(p_{z}-i p_{y}\right)^{2}+f_{5} .
\end{gathered}
$$

VI [41] $V=\alpha\left(z^{2}-2(x-i y)^{3}+4\left(x^{2}+y^{2}\right)\right)+\beta\left(2(x+i y)-3(x-i y)^{2}\right)+\gamma(x-i y)+\frac{\delta}{z^{2}}$,

$$
\begin{gathered}
\mathcal{S}_{1}=\left(p_{x}-i p_{y}\right)^{2}+f_{1}, \quad \mathcal{S}_{2}=p_{z}^{2}+f_{2}, \quad \mathcal{S}_{3}=p_{z}\left(J_{2}+i J_{1}\right)+f_{3}, \\
\mathcal{S}_{4}=J_{3}\left(p_{x}-i p_{y}\right)-\frac{i}{4}\left(p_{x}+i p_{y}\right)^{2}+f_{4}, \quad \mathcal{S}_{5}=\left(J_{2}+i J_{1}\right)^{2}+4 i p_{z} J_{1}+f_{5} .
\end{gathered}
$$$$
\text { VII [5] } \quad V=\alpha(x+i y)+\beta\left(\frac{3}{4}(x+i y)^{2}+\frac{1}{4} z\right)+\gamma\left((x+i y)^{3}+\frac{1}{16}(x-i y)+\frac{3}{4}(x+i y) z\right)
$$$$
+\delta\left(\frac{5}{16}(x+i y)^{4}+\frac{1}{16}\left(x^{2}+y^{2}+z^{2}\right)+\frac{3}{8}(x+i y)^{2} z\right) \text {, }
$$

$$
\begin{aligned}
& \mathcal{S}_{1}=\left(J_{1}+i J_{2}\right)^{2}+2 i J_{1}\left(p_{x}+i p_{y}\right)-J_{2}\left(p_{x}+i p_{y}\right)+\frac{1}{4}\left(p_{y}^{2}-p_{z}^{2}\right)-i J_{3} p_{z}+f_{1}, \\
& \mathcal{S}_{2}=J_{2} p_{z}-J_{3} p_{y}+i\left(J_{3} p_{x}-J_{1} p_{z}\right)-\frac{i}{2} p_{y} p_{z}+f_{2}, \quad \mathcal{S}_{3}=\left(p_{x}+i p_{y}\right)^{2}+f_{4}, \\
& \mathcal{S}_{4}=J_{3} p_{z}+i J_{1} p_{y}+i J_{2} p_{x}+2 J_{1} p_{x}+\frac{i}{4} p_{z}^{2}+f_{3}, \quad \mathcal{S}_{5}=p_{z}\left(p_{x}+i p_{y}\right)+f_{5} .
\end{aligned}
$$

Note that in the complete list of orthogonal separable coordinate systems for complex 3D Euclidean space there are some other systems besides the first seven that have parameter depen- 
dence, e.g., cylindrical elliptic coordinates $L_{1}=p_{3}^{2}, L_{2}=J_{3}^{2}+c^{2} p_{1}^{2}$. However, for all of these other coordinates the corresponding Bertrand-Darboux equations have only rank four, hence they do not uniquely determine a possible superintegrable system.

\section{Interbasis expansions for Euclidean systems}

To proceed with the classification of nondegenerate Euclidean superintegrable systems we need to look more closely at the relationship between a standard basis of symmetries for such a system and the natural basis written in terms of the linear and angular momentum generators $p_{k}, J_{k}, k=1, \ldots, 3$.

Let us denote our preferred Cartesian coordinate system by $\mathbf{x}=(u, v, w)$ and let $\mathbf{x}_{0}=(x, y, z)$, be a fixed regular point. We define the translated Cartesian coordinates $(X, Y, Z)$ by $u=x+X, v=y$ $+Y, w=z+Z$. Then, near the regular point $(x, y, z)$ we have a basis of "natural symmetries" $p_{1}$ $=p_{X}, p_{2}=p_{Y}, p_{3}=p_{Z}, J_{1}=Y p_{z}-Z p_{Y}, J_{2}=Z p_{X}-X p_{Z}, J_{3}=X p_{Y}-Y p_{X}$. Now suppose we have a Euclidean superintegrable system with nondegenerate potential. Then there will exist fifteen rational functions $A^{i j}(x, y, z), B^{i j}(x, y, z), C^{i j}(x, y, z)$, that completely characterize the superintegrable system. In particular, only 10 of these are linearly independent [see relations (A2)],

$$
A^{22}, A^{33}, B^{22}, B^{33}, C^{33}, A^{12}, B^{12}, A^{13}, A^{23}, B^{23},
$$

and they are subject to the five quadratic conditions (A3) with $G \equiv 0$. These functions are related to the symmetries $\mathcal{S}=\sum a^{i j} p_{i} p_{j}+W$ via the conditions (A1). Recall that the second order basis symmetries at the regular point $\mathcal{S}_{\mathbf{x}_{0}}^{(\ell m)}(\mathbf{x})=\sum a_{(\ell m)}^{i j}(\mathbf{x}) p_{i} p_{j}+f_{(\ell m)}$ take the form $\mathcal{S}_{\mathbf{x}_{0}}^{(\ell m)}\left(\mathbf{x}_{0}\right)=p_{i} p_{j}$ $+f_{(\ell m)}\left(\mathbf{x}_{0}\right)$ when evaluated at the point. Thus we can expand each standard basis symmetry in terms of the natural basis at the point via

$$
\begin{aligned}
\mathcal{S}_{\mathbf{x}_{0}}^{(\ell m)}= & p_{\ell} p_{m}+\alpha_{3}^{(\ell m)} J_{1}^{2}+\alpha_{4}^{(\ell m)} J_{2}^{2}+\alpha_{5}^{(\ell m)} J_{3}^{2}+\alpha_{6}^{(\ell m)} p_{1} J_{1}+\alpha_{7}^{(\ell m)} p_{2} J_{2}+\alpha_{8}^{(\ell m)} p_{1} J_{2}+\alpha_{9}^{(\ell m)} p_{1} J_{3} \\
& +\alpha_{10}^{(\ell m)} p_{2} J_{1}+\alpha_{11}^{(\ell m)} p_{2} J_{3}+\alpha_{12}^{(\ell m)} p_{3} J_{1}+\alpha_{13}^{(\ell m)} p_{3} J_{2}+\alpha_{14}^{(\ell m)} J_{1} J_{2}+\alpha_{15}^{(\ell m)} J_{1} J_{3} \\
& +\alpha_{16}^{(\ell m)} J_{2} J_{3}+W^{(\ell m)}(\mathbf{x}),
\end{aligned}
$$

where the $\alpha_{k}^{(\ell m)}$ are constants in $X, Y, Z$ but rational functions of the parameters $x, y, z$ of the regular point. [This notation for the expansion coefficients $\alpha_{s}$ is not completely logical, but since all of our software programs use the same notation we continue to use it to avoid (our) confusion.]

We conclude that all of the expansion constants $\alpha_{k}^{(\ell m)}$ can be expressed in terms of the ten numbers (23). However, we shall not embark on this straightforward task but instead restrict ourselves to expanding the two symmetries

$$
\begin{aligned}
\mathcal{S}_{\mathbf{x}_{0}}^{(12)}= & p_{1} p_{2}+\alpha_{3} J_{1}^{2}+\alpha_{4} J_{2}^{2}+\alpha_{5} J_{3}^{2}+\alpha_{6} p_{1} J_{1}+\alpha_{7} p_{2} J_{2}+\alpha_{8} p_{1} J_{2}+\alpha_{9} p_{1} J_{3}+\alpha_{10} p_{2} J_{1}+\alpha_{11} p_{2} J_{3} \\
& +\alpha_{12} p_{3} J_{1}+\alpha_{13} p_{3} J_{2}+\alpha_{14} J_{1} J_{2}+\alpha_{15} J_{1} J_{3}+\alpha_{16} J_{2} J_{3}+W^{(12)}(\mathbf{x}), \\
\mathcal{S}_{\mathbf{x}_{0}}^{(13)}= & p_{1} p_{3}+\alpha_{3}^{\prime} J_{1}^{2}+\alpha_{4}^{\prime} J_{2}^{2}+\alpha_{5}^{\prime} J_{3}^{2}+\alpha_{6}^{\prime} p_{1} J_{1}+\alpha_{7}^{\prime} p_{2} J_{2}+\alpha_{8}^{\prime} p_{1} J_{2}+\alpha_{9}^{\prime} p_{1} J_{3}+\alpha_{10}^{\prime} p_{2} J_{1}+\alpha_{11}^{\prime} p_{2} J_{3} \\
& +\alpha_{12}^{\prime} p_{3} J_{1}+\alpha_{13}^{\prime} p_{3} J_{2}+\alpha_{14}^{\prime} J_{1} J_{2}+\alpha_{15}^{\prime} J_{1} J_{3}+\alpha_{16}^{\prime} J_{2} J_{3}+W^{(13)}(\mathbf{x}) .
\end{aligned}
$$

(Here, $\alpha_{s}=\alpha_{s}^{(12)}, \alpha_{s}^{\prime}=\alpha_{s}^{(13)}$.) Indeed it is easy to verify that the six Bertrand-Darboux equations for these two symmetries have rank five (an illustration of Lemma 1 of Ref. 3). Thus these two symmetries completely determine the $A^{i j}, B^{i j}, C^{i j}$, hence the superintegrable system.

If $a^{i j}(\mathbf{x})$ is the quadratic form associated with $\mathcal{S}^{(12)}(\mathbf{x})$ it is straightforward to verify that

$$
\begin{aligned}
& a_{1}^{11}\left(\mathbf{x}_{0}\right)=0, \quad a_{2}^{11}\left(\mathbf{x}_{0}\right)=-\alpha_{9}, \quad a_{3}^{11}\left(\mathbf{x}_{0}\right)=\alpha_{8} \\
& a_{1}^{22}\left(\mathbf{x}_{0}\right)=\alpha_{11}, \quad a_{2}^{22}\left(\mathbf{x}_{0}\right)=0, \quad a_{3}^{22}\left(\mathbf{x}_{0}\right)=-\alpha_{10}
\end{aligned}
$$




$$
\begin{gathered}
a_{1}^{33}\left(\mathbf{x}_{0}\right)=-\alpha_{13}, \quad a_{2}^{33}\left(\mathbf{x}_{0}\right)=\alpha_{12}, \quad a_{3}^{33}\left(\mathbf{x}_{0}\right)=0, \\
a_{1}^{12}\left(\mathbf{x}_{0}\right)=\frac{1}{2} \alpha_{9}, \quad a_{2}^{12}\left(\mathbf{x}_{0}\right)=-\frac{1}{2} \alpha_{11}, \quad a_{3}^{12}\left(\mathbf{x}_{0}\right)=\frac{1}{2}\left(\alpha_{7}-\alpha_{6}\right), \\
a_{1}^{13}\left(\mathbf{x}_{0}\right)=-\frac{1}{2} \alpha_{8}, \quad a_{2}^{13}\left(\mathbf{x}_{0}\right)=\frac{1}{2} \alpha_{6}, \quad a_{3}^{13}\left(\mathbf{x}_{0}\right)=\frac{1}{2} \alpha_{13}, \\
a_{1}^{23}\left(\mathbf{x}_{0}\right)=-\frac{1}{2} \alpha_{7}, \quad a_{2}^{23}\left(\mathbf{x}_{0}\right)=\frac{1}{2} \alpha_{10}, \quad a_{3}^{23}\left(\mathbf{x}_{0}\right)=-\frac{1}{2} \alpha_{12},
\end{gathered}
$$

where $a_{k}^{i j}\left(\mathbf{x}_{0}\right)=\left.\partial_{k} a^{i j}(\mathbf{x})\right|_{\mathbf{x}_{0}}$. There are identical relations for the other symmetries $\mathcal{S}^{(\ell m)}(\mathbf{x})$. Using (27) and the identities (A2) and (A1) we can express the expansion coefficients $\alpha_{6}, \ldots, \alpha_{13}$ in terms of the ten numbers (23) at $\mathbf{x}_{0}$ :

$$
\begin{gathered}
\alpha_{6}=\frac{1}{3}\left(2 A^{13}-B^{23}\right), \quad \alpha_{7}=\frac{1}{3}\left(A^{13}-2 B^{23}\right), \quad \alpha_{8}=-\frac{1}{3} A^{23}, \\
\alpha_{9}=\frac{1}{3} A^{22}, \quad \alpha_{10}=\frac{1}{3} A^{23}, \quad \alpha_{11}=\frac{1}{3} B^{22}, \\
\alpha_{12}=\frac{1}{3}\left(B^{12}-A^{22}+A^{33}\right), \quad \alpha_{13}=-\frac{1}{3}\left(B^{33}+A^{12}\right) .
\end{gathered}
$$

The corresponding results for the expansion coefficients $\alpha_{6}^{\prime}, \ldots, \alpha_{13}^{\prime}$ of $\mathcal{S}^{(13)}$ are

$$
\begin{gathered}
\alpha_{6}^{\prime}=-\frac{1}{3}\left(2 A^{12}+B^{33}\right), \quad \alpha_{7}^{\prime}=-\frac{1}{3}\left(A^{12}+2 B^{23}\right), \quad \alpha_{8}^{\prime}=-\frac{1}{3} A^{33}, \\
\alpha_{9}^{\prime}=\frac{1}{3} A^{23}, \quad \alpha_{10}^{\prime}=-\frac{1}{3} B^{12}, \quad \alpha_{11}^{\prime}=\frac{1}{3} B^{23}, \\
\alpha_{12}^{\prime}=-\frac{1}{3} A^{23}, \quad \alpha_{13}^{\prime}=-\frac{1}{3} C^{33} .
\end{gathered}
$$

The expansion coefficients of the terms of the form $J_{\ell} J_{m}$, i.e., $\alpha_{3}, \alpha_{4}, \alpha_{5}, \alpha_{14}, \alpha_{15}, \alpha_{16}$ can be expressed in terms of second derivatives of the associated quadratic form, evaluated at the regular point $\mathbf{x}_{0}$. For example, $\alpha_{14}=2 a_{23}^{13}\left(\mathbf{x}_{0}\right)=-a_{33}^{12}\left(\mathbf{x}_{0}\right)=-a_{12}^{33}\left(\mathbf{x}_{0}\right)$. For a superintegrable system the integrability conditions for the symmetry relations (A1) are satisfied identically, so these equations can be differentiated to compute the second derivatives $a_{k \ell}^{i j}\left(\mathbf{x}_{0}\right)$ as a quadratic expression in the ten basic constants [subject to the five quadratic identities (A3)]. Though straightforward, these computations are tedious. The only relations that we will use here are those for the expansion coefficients $\alpha_{14}^{(\ell m)}$. We have

$$
\begin{gathered}
\alpha_{14}^{(11)}=\frac{1}{9}\left(4 A^{23}\left(B^{33}-B^{22}\right)-4 B^{23}\left(A^{23}-A^{22}\right)-2 A^{13} B^{12}+2 A^{12} A^{23}\right) \\
\alpha_{14}^{(22)}=\frac{1}{9}\left(4 A^{12} A^{23}+2 B^{12} B^{23}+2 A^{23} B^{33}-2 A^{13} B^{12}-2 B^{23} A^{33}+2 B^{23} A^{22}-4 B^{12} B^{23}\right) \\
\alpha_{14}^{(33)}=\frac{1}{9}\left(2 B^{23}\left(A^{22}-A^{33}+B^{12}\right)-4 A^{13} B^{12}+2 A^{23}\left(A^{12}-2 B^{22}+B^{33}\right)\right) \\
\alpha_{14}^{(12)}=\frac{1}{9}\left(\left(2 B^{23}-A^{13}\right)\left(B^{33}-B^{22}\right)-2\left(B^{33}+A^{12}-B^{22}\right) B^{23}\right. \\
\left.-A^{23} B^{12}+\left(2 B^{23}+A^{13}\right) A^{12}+2 B^{33} A^{13}-A^{23} A^{33}\right), \\
\alpha_{14}^{(13)}=\frac{1}{18}\left(7\left(B^{33}\right)^{2}+\left(A^{33}\right)^{2}-2 A^{22} B^{12}-\left(A^{23}\right)^{2}+4 A^{12} B^{33}-A^{13} C^{33}-3\left(A^{12}\right)^{2}-5\left(B^{12}\right)^{2}-4 A^{33} B^{12}\right. \\
\left.-7 B^{22} B^{33}-7 B^{23} C^{33}+2 A^{13} B^{23}+7\left(B^{23}\right)^{2}-A^{22} A^{33}\right),
\end{gathered}
$$




$$
\alpha_{14}^{(23)}=\frac{1}{9}\left(A^{23}\left(-B^{23}+C^{33}\right)+\left(A^{23}-A^{22}+B^{12}\right)\left(A^{12}+B^{22}-B^{33}\right)\right) .
$$

Note that since the Hamiltonian is $\mathcal{S}^{(11)}+\mathcal{S}^{(22)}+\mathcal{S}^{(33)}$ and the coefficient of $J_{1} J_{2}$ in the Hamiltonian is 0 , we must have $\alpha_{14}^{(11)}+\alpha_{14}^{(22)}+\alpha_{14}^{(33)}=0$, which can be verified directly from the above-mentioned expressions.

As a result of the previous discussion we have the result

Theorem 6: For a nondegenerate superintegrable system the expansion coefficients $\alpha_{k}^{(\ell m)}$ expressing the standard basis $\mathcal{S}^{\ell m}$ in terms of the natural basis $p_{h} p_{k}, p_{h} J_{k}, J_{h} J_{k}$ are explicit linear and quadratic expressions in the ten terms (23).

\section{The significance of generic Euclidean systems}

Suppose we have a nondegenerate Euclidean superintegrable system with potential $V$ that is separable with respect to some orthogonal coordinates. (Since every superintegrable system is multiseparable, we know that such coordinates exist.) By performing an Euclidean transformation, if necessary, we can assume that the separable coordinates are in a standard form determined by two constants of the motion in involution,

$$
L_{1}=\sum a^{i j} p_{i} p_{j}+f_{1}, \quad L_{2}=\sum b^{i j} p_{i} p_{j}+f_{2} .
$$

Clearly, $L_{1}$ and $L_{2}$ lie in the six-dimensional space of second order symmetries for the superintegrable system. Thus, the quadratic form $a^{i j}$, for example, satisfies the three Bertrand-Darboux equations for potential $V$. Since $V$ is nondegenerate we can express the second derivatives $V_{j j}$ $-V_{k k}$ and $V_{j k}$ with $j \neq k$ in the Bertrand-Darboux equations as linear combinations of the first derivatives $V_{h}$. Equating coefficients of $V_{1}, V_{2}, V_{3}$ separately in each of the three equations, we end up with nine linear conditions for the ten constants $A^{22}, \ldots, B^{23}$ at each regular point. A typical example of one of these conditions is

$$
\begin{aligned}
& A^{13}\left(3 a^{11}-3 a^{33}\right)+B^{23}(0)+A^{23}\left(-3 a^{12}\right)+A^{22}(0)+B^{22}(0)+B^{12}(0)+A^{33}\left(-3 a^{13}\right)+B^{33}(0) \\
& \quad+A^{12}\left(-3 a^{23}\right)+C^{33}(0)=-a_{1}^{13}+a_{3}^{11} .
\end{aligned}
$$

Here, $B^{23}(0)=0$, etc. For the second symmetry there will be nine more linear conditions with $a^{i j}$ replaced by $b^{i j}$. Thus we will have eighteen linear equations (not linearly independent) for the ten quantities $A^{22}, \ldots, B^{23}$. Another source of conditions is obtained by writing the symmetry $L_{1}$ in terms of the standard basis:

$$
a^{i j}(\mathbf{x})=\sum_{\ell \leqslant m} a^{\ell m}\left(\mathbf{x}_{0}\right) \mathcal{A}_{(\ell, m)}^{i j}(\mathbf{x}),
$$

where $\mathcal{A}_{(\ell, m)}^{i j}$ is the quadratic form associated with the standard basis symmetry $\mathcal{S}^{(\ell, m)}$ at $\mathbf{x}_{0}$. Expanding both sides of this equation in terms of the natural basis we obtain linear and quadratic conditions on the ten basic quantities. For example if we equate coefficients of the natural basis element $J_{1} J_{2}$ we find the quadratic conditions for $L_{1}$ and $L_{2}$ :

$$
2 a_{23}^{13}\left(\mathbf{x}_{0}\right)=\sum_{\ell \leqslant m} a^{\ell m}\left(\mathbf{x}_{0}\right) \alpha_{14}^{(\ell m)}, \quad 2 b_{23}^{13}\left(\mathbf{x}_{0}\right)=\sum_{\ell \leqslant m} b^{\ell m}\left(\mathbf{x}_{0}\right) \alpha_{14}^{(\ell m)} .
$$

Though there are many other quadratic conditions for $L_{1}, L_{2}$ to belong to the symmetry algebra, we shall use only these two and the five fundamental quadratic identities (A3) that hold independent of any choice of $L_{1}, L_{2}$. Note that by equating coefficients of natural basis elements of the form $p_{i} J_{k}$ we could obtain linear identities. However, these are equivalent to the linear conditions for $a^{i j}, b^{i j}$ already discussed previously.

We give an example to show how this works. Suppose we have a nondegenerate superintegrable system that admits separation for some special choice of ellipsoidal coordinates [2111]. (Here we do not assume that the system separates for all values of the parameters $c, e_{1}, e_{2}, e_{3}$, but only for one value.) By performing an Euclidean transformation and a change of scale we can 
assume that the coordinates are in the standard form [2111] in our table and that $c=1, e_{1}=0, e_{2}$ $=1$, and $e_{3}=a$ where $a$ is any fixed complex number such that $a(a-1) \neq 0$. It follows that

$$
\begin{aligned}
& a^{11}=y^{2}+z^{2}+a+1, \quad a^{22}=x^{2}+z^{2}+a, \quad a^{33}=x^{2}+y^{2}+1, \\
& a^{12}=-x y, \quad a^{13}=-x z, \quad a^{23}=-y z, \quad b^{11}=a y^{2}+z^{2}+a, \\
& b^{22}=a x^{2}, \quad b^{33}=x^{2}, \quad b^{12}=-a x y, \quad b^{13}=-x z, \quad b^{23}=0,
\end{aligned}
$$

at any regular point with coordinates $(x, y, z)$. Substituting these expressions into the 18 linear conditions discussed previously, with the help of the computer algebra system MAPLE, we find that there are exactly seven independent linear conditions. Thus the ten quantities $A^{22}, \ldots, B^{23}$ can be expressed linearly in terms of three of these quantities. Substituting this result into the five fundamental quadratic identities (A3) we find that these identities yield a single linear relation for the remaining three unknowns. Finally we substitute our expressions in terms of the three unknowns and (30) into (50) and obtain (with the help of MAPLE) two more independent linear conditions. Thus we end up with ten independent linear conditions for our ten unknowns, and we obtain the unique solution

$$
A^{12}=B^{12}=A^{23}=B^{23}=A^{13}=B^{33}=0, \quad A^{33}=A^{22}=\frac{3}{x}, \quad C^{33}=-\frac{3}{z}, \quad B^{22}=-\frac{3}{y},
$$

which corresponds to the nondegenerate potential [I],

$$
V=\frac{\alpha}{x^{2}}+\frac{\beta}{y^{2}}+\frac{\gamma}{z^{2}}+\delta\left(x^{2}+y^{2}+z^{2}\right) .
$$

Note that it was obvious that our conditions would have solutions, since we already knew that system [I] separated simultaneously for all choices of the parameters $c, e_{1}, e_{2}, e_{3}$. What was far from obvious is the fact that no other nondegenerate superintegrable system separates for any special case of ellipsoidal coordinates.

Theorem 7: A 3D Euclidean nondegenerate superintegrable system admits separation in a special case of the generic coordinates [2111], [221], [23], [311], [32], [41], or [5], respectively, if and only if it is equivalent via a Euclidean transformation to system [I], [II], [III], [IV], [V], [VI], or [VII], respectively.

The proof (complicated but straightforward) proceeds exactly as the case [2111] described previously. For each case [221]-[5] we use the symmetries $a^{i j}, b^{i j}$ listed. The eighteen linear conditions discussed previously reduce to exactly seven independent linear conditions. Thus always the ten quantities $A^{22}, \ldots, B^{23}$ can be expressed linearly in terms of three of these quantities. Substituting into the five fundamental quadratic identities (A3) we find that these identities yield a single linear relation for the remaining three unknowns. Substituting our expressions in terms of the three unknowns and (30) into (50) we obtain two more independent linear conditions. Thus we end up with ten independent linear conditions for our ten unknowns, and a unique solution, the corresponding generic superintegrable system.

This does not settle the problem of classifying all 3D nondegenerate superintegrable systems in complex Euclidean space, for we have not excluded the possibility of such systems that separate only in degenerate separable coordinates. In fact we have already studied two such systems in: ${ }^{3}$

$$
\begin{gathered}
\text { [O] } V(x, y, z)=\alpha x+\beta y+\gamma z+\delta\left(x^{2}+y^{2}+z^{2}\right) \\
{[O O] \quad V(x, y, z)=\frac{\alpha}{2}\left(x^{2}+y^{2}+\frac{1}{4} z^{2}\right)+\beta x+\gamma y+\frac{\delta}{z^{2}} .}
\end{gathered}
$$


However, both of these nondegenerate superintegrable systems are only weakly functionally independent, in contrast to systems [I]-[VII]. Thus we consider $[O]$ and $[O O]$ as associate members of the superintegrable family, not regular members. An investigation of other possible Euclidean systems is in progress.

\section{E. Generic superintegrable systems on the three sphere}

An important task remaining is to classify the possible systems on the three sphere (particularly those three-sphere systems not Stäckel equivalent to a flat space system). We choose a standardized Cartesian-like coordinate system $\{x, y, z\}$ on the three sphere such that the metric and Hamiltonian are

$$
\mathrm{d} s^{2}=\frac{1}{\left(1+\frac{r^{2}}{4}\right)^{2}}\left(\mathrm{~d} x^{2}+\mathrm{d} y^{2}+\mathrm{d} z^{2}\right), \quad \mathcal{H}=\left(1+\frac{r^{2}}{4}\right)^{2}\left(p_{x}^{2}+p_{y}^{2}+p_{z}^{2}\right)+V,
$$

where $r^{2}=x^{2}+y^{2}+z^{2}$. These coordinates can be related to the standard realization of the sphere via complex coordinates $\mathbf{s}=\left(s_{1}, s_{2}, s_{3}, s_{4}\right)$ such that $\sum_{j=1}^{4} s_{j}^{2}=1$ and $\mathrm{d} s^{2}=\Sigma_{j} \mathrm{~d} s_{j}^{2}$ via

$$
s_{1}=\frac{4 x}{4+r^{2}}, \quad s_{2}=\frac{4 y}{4+r^{2}}, \quad s_{3}=\frac{4 z}{4+r^{2}}, \quad s_{4}=\frac{4-r^{2}}{4+r^{2}}
$$

with inverse $x=2 s_{1} /\left(1+s_{4}\right), y=2 s_{2} /\left(1+s_{4}\right), z=2 s_{3} /\left(1+s_{4}\right)$. Here $x, y, z$ are local coordinates in a neighborhood of the pole $\mathbf{P}=(0,0,0,1)$ on the three sphere. A basis of Killing vectors for the zero potential system is $J_{h}, K_{h}, h=1,2,3$ where

$$
\begin{gathered}
J_{1}=y p_{z}-z p_{y}, \quad J_{2}=z p_{x}-x p_{z}, \quad J_{3}=x p_{y}-y p_{x}, \\
K_{1}=\left(1+\frac{x^{2}-y^{2}-z^{2}}{4}\right) p_{x}+\frac{x y}{2} p_{y}+\frac{x z}{2} p_{z}, \quad K_{2}=\left(1+\frac{y^{2}-x^{2}-z^{2}}{4}\right) p_{y}+\frac{x y}{2} p_{x}+\frac{y z}{2} p_{z}, \\
K_{3}=\left(1+\frac{z^{2}-x^{2}-y^{2}}{4}\right) p_{z}+\frac{x z}{2} p_{x}+\frac{y z}{2} p_{y} .
\end{gathered}
$$

The commutation relations are

$$
\left\{J_{1}, J_{2}\right\}=J_{3}, \quad\left\{K_{1}, K_{2}\right\}=J_{3}, \quad\left\{K_{1}, J_{2}\right\}=K_{3}
$$

and their cyclic permutations. The relation between this basis and the standard basis of rotation generators on the sphere $I_{\ell m}=s_{\ell} p_{m}-s_{m} p_{\ell}=-I_{m \ell}$ is

$$
J_{1}=I_{23}, \quad J_{2}=I_{31}, \quad J_{3}=I_{12}, \quad K_{1}=I_{41}, \quad K_{2}=I_{42}, \quad K_{3}=I_{43} .
$$

We shall use the $x, y, z$ coordinates as standard but we also need to see how these coordinates relate to analogous Cartesian-like coordinates centered at any point $\mathbf{T}$ on the sphere. We can always find a complex orthogonal matrix $O$, not unique, such that $\mathbf{T}=O \mathbf{P}$. If $X, Y, Z$, (34), define local Cartesian-like coordinates near $\mathbf{P}$ then via $\mathbf{t}=O s(X, Y, Z)$ they also define local coordinates in a neighborhood of $\mathbf{T}=\left(T_{1}, T_{2}, T_{3}, T_{4}\right)$. Moreover, since $O$ is orthogonal we have

$$
\mathrm{d} s^{2}=\mathrm{d} \mathbf{t} \cdot \mathrm{d} \mathbf{t}=\mathrm{d} O \mathbf{s} \cdot \mathrm{d} O \mathbf{s}=\mathrm{d} \mathbf{s} \cdot \mathrm{d} \mathbf{s}=\frac{1}{\left(1+\frac{R^{2}}{4}\right)^{2}}\left(\mathrm{~d} X^{2}+\mathrm{d} Y^{2}+\mathrm{d} Z^{2}\right),
$$

so we can consider $X, Y, Z$ as Cartesian-like coordinates in a neighborhood of $\mathbf{T}$. We can also require that the coordinate axes line up so that differentiation of $\mathbf{s}$ by $X, Y, Z$, respectively, at $\mathbf{P}$ corresponds to (normalized) differentiation of $\mathbf{t}$ by $x, y, z$, respectively, at $\mathbf{T}$, i.e., so that $p_{X}$ corresponds to $\left(1+r^{2} / 4\right) p_{x}$, etc. Thus, 


$$
\begin{gathered}
\left.\left(\frac{2}{1+t_{4}} \partial_{x} \mathbf{t}\right)\right|_{\mathbf{t}=\mathbf{T}}=O\left(\begin{array}{l}
1 \\
0 \\
0 \\
0
\end{array}\right),\left.\quad\left(\frac{2}{1+t_{4}} \partial_{y} \mathbf{t}\right)\right|_{\mathbf{t}=T}=O\left(\begin{array}{l}
0 \\
1 \\
0 \\
0
\end{array}\right), \\
\left.\left(\frac{2}{1+t_{4}} \partial_{z} \mathbf{t}\right)\right|_{\mathbf{t}=T}=O\left(\begin{array}{l}
0 \\
0 \\
1 \\
0
\end{array}\right), \quad \mathbf{T}=O\left(\begin{array}{l}
0 \\
0 \\
0 \\
1
\end{array}\right) .
\end{gathered}
$$

This determines $O$ uniquely, since the column vectors on the left-hand sides of these expressions are mutually orthogonal unit vectors. We find

$$
O_{T}=\left(\begin{array}{cccc}
-\frac{T_{1}^{2}-T_{4}-1}{T_{4}+1} & -\frac{T_{2} T_{1}}{T_{4}+1} & -\frac{T_{3} T_{1}}{T_{4}+1} & T_{1} \\
-\frac{T_{1} T_{2}}{T_{4}+1} & -\frac{T_{2}^{2}-T_{4}-1}{T_{4}+1} & -\frac{T_{3} T_{2}}{T_{4}+1} & T_{2} \\
-\frac{T_{1} T_{3}}{T_{4}+1} & -\frac{T_{2} T_{3}}{T_{4}+1} & -\frac{T_{3}^{2}-T_{4}-1}{T_{4}+1} & T_{3} \\
-T_{1} & -T_{2} & -T_{3} & T_{4}
\end{array}\right) .
$$

In the P-based coordinate system the coordinates of $\mathbf{t}$ are $u, v, w$ where $u=2 t_{1} /\left(1+t_{4}\right), v$ $=2 t_{2} /\left(1+t_{4}\right), w=2 t_{3} /\left(1+t_{4}\right)$. From the equation $\mathbf{t}=O_{T} \mathbf{s}$ we can solve for $u, v, w$ to obtain

$$
\begin{aligned}
& u=\frac{4\left[r^{2} X-2 x(x X+y Y+z Z)+4(x+X)-x R^{2}\right]}{16-8(x X+y Y+z Z)+r^{2} R^{2}}, \\
& v=\frac{4\left[r^{2} Y-2 y(x X+y Y+z Z)+4(y+Y)-y R^{2}\right]}{16-8(x X+y Y+z Z)+r^{2} R^{2}}, \\
& w=\frac{4\left[r^{2} Z-2 z(x X+y Y+z Z)+4(z+Z)-z R^{2}\right]}{16-8(x X+y Y+z Z)+r^{2} R^{2}} .
\end{aligned}
$$

To recapitulate: $\mathbf{t}$ is a point on the complex unit sphere, $(x, y, z)$ are the coordinates of $\mathbf{T}$ in the $\mathbf{P}$-based system, $(u, v, w)$ are the coordinates of $\mathbf{t}$ in the $\mathbf{P}$-based system, and $(X, Y, Z)$ are the coordinates of $\mathbf{t}$ in the $\mathbf{T}$-based system. Thus, for fixed $\mathbf{T}$, Eq. (39) defines the coordinate transformation between $(u, v, w)$ and $(X, Y, Z)$. We can write Eq. (39) in a simpler form by introducing the supplementary variables

$$
U=\frac{u-x}{1+\frac{r^{2}}{4}}, \quad V=\frac{v-y}{1+\frac{r^{2}}{4}}, \quad W=\frac{v-z}{1+\frac{r^{2}}{4}}, \quad Q^{2}=U^{2}+V^{2}+W^{2}
$$

Then

$$
U=\frac{1-\frac{1}{2} x X}{1-\frac{1}{2}(x X+y Y+z Z)+\frac{r^{2} R^{2}}{16}},
$$




$$
V=\frac{1-\frac{1}{2} y Y}{1-\frac{1}{2}(x X+y Y+z Z)+\frac{r^{2} R^{2}}{16}}, \quad W=\frac{1-\frac{1}{2} z Z}{1-\frac{1}{2}(x X+y Y+z Z)+\frac{r^{2} R^{2}}{16}},
$$

with inverse

$$
\begin{gathered}
X=\frac{U+\frac{x}{4} Q^{2}}{1+\frac{1}{2}(x U+y V+z W)+\frac{r^{2} Q^{2}}{16}}, \\
Y=\frac{V+\frac{y}{4} Q^{2}}{1+\frac{1}{2}(x U+y V+z W)+\frac{r^{2} Q^{2}}{16}}, \quad Z=\frac{W+\frac{z}{4} Q^{2}}{1+\frac{1}{2}(x U+y V+z W)+\frac{r^{2} Q^{2}}{16}} .
\end{gathered}
$$

In Ref. 22 we have determined all orthogonal separable coordinate systems on the complex unit three sphere. Of the 21 systems listed those that are generic, in the sense we used for Euclidean separable systems, are given as follows with coordinates followed by defining constants of the motion. (Here we take the Hamiltonian as $\mathcal{L}_{0}=I_{12}^{2}+I_{13}^{2}+I_{14}^{2}+I_{23}^{2}+I_{24}^{2}+I_{34}^{2}$, and we recall the identity $I_{23} I_{41}+I_{31} I_{42}+I_{12} I_{43}=0$.)

[1111] (system (17) in Ref. [22])

$$
\begin{gathered}
s_{1}^{2}=\frac{\left(x_{1}-e_{1}\right)\left(x_{2}-e_{1}\right)\left(x_{3}-e_{1}\right)}{\left(e_{1}-e_{2}\right)\left(e_{1}-e_{3}\right)\left(e_{1}-e_{4}\right)}, \quad s_{2}^{2}=\frac{\left(x_{1}-e_{2}\right)\left(x_{2}-e_{2}\right)\left(x_{3}-e_{2}\right)}{\left(e_{2}-e_{1}\right)\left(e_{2}-e_{3}\right)\left(e_{2}-e_{4}\right)}, \\
s_{3}^{2}=\frac{\left(x_{1}-e_{3}\right)\left(x_{2}-e_{3}\right)\left(x_{3}-e_{3}\right)}{\left(e_{3}-e_{1}\right)\left(e_{3}-e_{2}\right)\left(e_{3}-e_{4}\right)}, \quad s_{4}^{2}=\frac{\left(x_{1}-e_{4}\right)\left(x_{2}-e_{4}\right)\left(x_{3}-e_{4}\right)}{\left(e_{4}-e_{2}\right)\left(e_{4}-e_{3}\right)\left(e_{4}-e_{1}\right)}, \\
\mathcal{L}_{1}=\left(e_{1}+e_{2}\right) I_{12}^{2}+\left(e_{1}+e_{3}\right) I_{13}^{2}+\left(e_{1}+e_{4}\right) I_{14}^{2}+\left(e_{2}+e_{3}\right) I_{23}^{2}+\left(e_{2}+e_{4}\right) I_{24}^{2}+\left(e_{3}+e_{4}\right) I_{34}^{2}, \\
\mathcal{L}_{2}=e_{1} e_{2} I_{12}^{2}+e_{1} e_{3} I_{13}^{2}+e_{1} e_{4} I_{14}^{2}+e_{2} e_{3} I_{23}^{2}+e_{2} e_{4} I_{24}^{2}+e_{3} e_{4} I_{34}^{2} .
\end{gathered}
$$

[211] (system (18) in Ref. [22])

$$
\begin{gathered}
\left(i s_{1}+s_{2}\right)^{2}=-2 \frac{\left(x_{1}-e_{1}\right)\left(x_{2}-e_{1}\right)\left(x_{3}-e_{1}\right)}{\left(e_{1}-e_{3}\right)\left(e_{1}-e_{4}\right)}, \\
s_{1}^{2}+s_{2}^{2}=-\partial_{e_{1}}\left(\frac{\left(x_{1}-e_{1}\right)\left(x_{2}-e_{1}\right)\left(x_{3}-e_{1}\right)}{\left(e_{1}-e_{3}\right)\left(e_{1}-e_{4}\right)}\right), \\
s_{3}^{2}=-\frac{\left(x_{1}-e_{3}\right)\left(x_{2}-e_{3}\right)\left(x_{3}-e_{3}\right)}{\left(e_{3}-e_{1}\right)^{2}\left(e_{3}-e_{4}\right)}, \quad s_{4}^{2}=-\frac{\left(x_{1}-e_{4}\right)\left(x_{2}-e_{4}\right)\left(x_{3}-e_{4}\right)}{\left(e_{4}-e_{1}\right)^{2}\left(e_{4}-e_{3}\right)} ; \\
\mathcal{L}_{1}=\left(I_{14}+i I_{24}\right)^{2}+\left(I_{13}+i I_{23}\right)^{2}+2 e_{1}\left(2 I_{12}^{2}+I_{14}^{2}+I_{24}^{2}+I_{13}^{2}+I_{23}^{2}\right)+2 e_{3}\left(I_{34}^{2}+I_{13}^{2}+I_{23}^{2}\right) \\
+2 e_{4}\left(I_{14}^{2}+I_{24}^{2}+I_{34}^{2}\right), \\
\mathcal{L}_{2}=e_{1}^{2} I_{12}^{2}+e_{1} e_{3}\left(I_{13}^{2}+I_{23}^{2}\right)+e_{1} e_{4}\left(I_{14}^{2}+I_{24}^{2}\right)+e_{3} e_{4} I_{34}^{2}+\frac{e_{3}}{2}\left(I_{13}+i I_{23}\right)^{2}+\frac{e_{4}}{2}\left(I_{14}+i I_{24}\right)^{2} .
\end{gathered}
$$

[22] (system (19) in Ref. [22]) 


$$
\begin{gathered}
\left(s_{1}+i s_{2}\right)^{2}=-2 \frac{\left(x_{1}-e_{1}\right)\left(x_{2}-e_{1}\right)\left(x_{3}-e_{1}\right)}{\left(e_{1}-e_{3}\right)^{2}}, \\
\left(s_{3}+i s_{4}\right)^{2}=-2 \frac{\left(x_{1}-e_{3}\right)\left(x_{2}-e_{3}\right)\left(x_{3}-e_{3}\right)}{\left(e_{1}-e_{3}\right)^{2}}, \quad s_{1}^{2}+s_{2}^{2}=-\frac{\partial}{\partial e_{1}}\left(\frac{\left(x_{1}-e_{1}\right)\left(x_{2}-e_{1}\right)\left(x_{3}-e_{1}\right)}{\left(e_{1}-e_{3}\right)^{2}}\right) ; \\
\mathcal{L}_{1}=-I_{24}^{2}+I_{13}^{2}+i I_{13} I_{23}+i I_{14} I_{24}+i I_{23} I_{24}+i I_{13} I_{14}+\left(e_{1}-e_{3}\right)\left[I_{12}^{2}-I_{34}^{2}\right]+e_{2}, \\
\mathcal{L}_{2}=e_{1}^{2} I_{12}^{2}+e_{3}^{2} I_{34}^{2}+e_{1} e_{3}\left[I_{13}^{2}+I_{24}^{2}+I_{14}^{2}+I_{23}^{2}\right]+\frac{1}{4}\left[I_{13}^{2}+I_{24}^{2}-I_{14}^{2}-I_{23}^{2}+2 i I_{13} I_{23}+2 i I_{13} I_{14}-2 i I_{14} I_{24}\right. \\
\left.-2 i I_{23} I_{24}-4 I_{13} I_{24}-2 I_{12} I_{34}\right]+\frac{e_{1}}{2}\left[I_{13}^{2}-I_{24}^{2}-I_{14}^{2}+I_{23}^{2}+2 i I_{13} I_{14}+2 i I_{23} I_{24}\right]+\frac{e_{3}}{2}\left[-I_{24}^{2}+I_{13}^{2}+I_{14}^{2}\right. \\
\left.-I_{23}^{2}+2 i I_{13} I_{23}+2 i I_{14} I_{24}\right] .
\end{gathered}
$$

[31] (system (20) in Ref. in [22])

$$
\begin{gathered}
\left(s_{1}+i s_{2}\right)^{2}=-2 \frac{\left(x_{1}-e_{1}\right)\left(x_{2}-e_{1}\right)\left(x_{3}-e_{1}\right)}{\left(e_{1}-e_{4}\right)}, \quad s_{4}^{2}=-\frac{\left(x_{1}-e_{4}\right)\left(x_{2}-e_{4}\right)\left(x_{3}-e_{4}\right)}{\left(e_{4}-e_{1}\right)^{3}}, \\
\sqrt{2} s_{3}\left(s_{1}+i s_{2}\right)=-\frac{\partial}{\partial e_{1}}\left(\frac{\left(x_{1}-e_{1}\right)\left(x_{2}-e_{1}\right)\left(x_{3}-e_{1}\right)}{\left(e_{1}-e_{4}\right)}\right) \\
s_{1}^{2}+s_{2}^{2}+s_{3}^{2}=-\frac{1}{2} \frac{\partial^{2}}{\partial e_{1}^{2}} \frac{\left(x_{1}-e_{1}\right)\left(x_{2}-e_{1}\right)\left(x_{3}-e_{1}\right)}{\left(e_{1}-e_{4}\right)} ; \\
\mathcal{L}_{1}=\sqrt{2}\left(I_{14} I_{34}-I_{12} I_{23}+i I_{24} I_{34}+i I_{12} I_{13}\right)+e_{1}\left(I_{12}^{2}+I_{13}^{2}+I_{23}^{2}\right)+e_{4}\left(I_{34}^{2}+I_{14}^{2}+I_{24}^{2}\right), \\
\mathcal{L}_{2}=-\frac{1}{2} I_{13}^{2}+\frac{1}{2} I_{23}^{2}-i I_{13} I_{23}+e_{1} e_{4}\left(I_{34}^{2}+I_{14}^{2}+I_{24}^{2}\right)=e_{1}^{2}\left(I_{12}^{2}+I_{13}^{2}+I_{23}^{2}\right)-\frac{e_{1}}{\sqrt{2}}\left(-2 i I_{12} I_{13}+2 I_{12} I_{23}\right) \\
+\sqrt{2} e_{4}\left(I_{14} I_{34}+i I_{24} I_{34}\right) .
\end{gathered}
$$

[4] (system (21) in [22])

$$
\begin{gathered}
\left(s_{1}+i s_{2}\right)^{2}=-2\left(x_{1}-e_{1}\right)\left(x_{2}-e_{2}\right)\left(x_{3}-e_{3}\right), \\
\left(s_{1}+i s_{2}\right)\left(s_{3}+i s_{4}\right)=-\frac{\partial}{\partial e_{1}}\left(\left(x_{1}-e_{1}\right)\left(x_{2}-e_{1}\right)\left(x_{3}-e_{1}\right)\right), \\
2\left(s_{1}+i s_{2}\right)\left(s_{3}-i s_{4}\right)+\left(s_{3}+i s_{4}\right)^{2}=-\frac{\partial^{2}}{\partial e_{1}^{2}}\left(\left(x_{1}-e_{1}\right)\left(x_{2}-e_{1}\right)\left(x_{3}-e_{1}\right)\right) ; \\
\mathcal{L}_{1}=\frac{1}{2}\left(2 I_{34} I_{14}+2 I_{12} I_{14}-2 I_{23} I_{34}-2 I_{12} I_{23}-I_{24}^{2}+I_{23}^{2}-I_{14}^{2}+I_{13}^{2}+2 i I_{13} I_{14}+2 i I_{23} I_{24}+2 i I_{12} I_{24}\right. \\
\left.+2 i I_{13} I_{34}+2 i I_{24} I_{34}+2 i I_{12} I_{13}\right),
\end{gathered}
$$




$$
\begin{aligned}
\mathcal{L}_{2}= & -I_{14}^{2}-I_{23} I_{34}-\frac{1}{4} I_{24}^{2}+\frac{1}{4} I_{23}^{2}+\frac{1}{4} I_{14}^{2}-\frac{1}{4} I_{13}^{2}-\frac{i}{2} I_{13} I_{23}-\frac{i}{2} I_{23} I_{24} \frac{i}{2} I_{14} I_{24}+\frac{i}{2} I_{13} I_{14}-i I_{24} I_{34}+i I_{13} I_{34} \\
& -\frac{1}{2} I_{13} I_{34}-\frac{1}{2} I_{14} I_{23} .
\end{aligned}
$$

We now show that each generic separable system on the three sphere uniquely determines a superintegrable system with nondegenerate potential. The proof is, in most part, analogous to that for the Euclidean case. Consider system (1111). If we have a superintegrable system that admits the symmetries $\mathcal{L}_{1}, \mathcal{L}_{2}$ for all values of the parameters $e_{1}, \ldots, e_{4}$ then it must have the basis of symmetries

$$
\begin{gathered}
\text { VIII } \mathcal{S}_{0}=I_{12}^{2}+f_{0}, \quad \mathcal{S}_{1}=I_{13}^{2}+f_{1}, \quad \mathcal{S}_{2}=I_{14}^{2}+f_{2}, \quad \mathcal{S}_{3}=I_{32}^{2}+f_{3}, \\
\mathcal{S}_{4}=I_{24}^{2}+f_{4}, \quad \mathcal{S}_{5}=I_{34}^{2}+f_{5} .
\end{gathered}
$$

The system of Bertrand-Darboux equations associated with these symmetries has rank five so the potential is uniquely determined. Solving the Bertrand-Darboux equations we obtain the nondegenerate potential on the three sphere

$$
V(s)=\frac{\alpha}{s_{1}^{2}}+\frac{\beta}{s_{2}^{2}}+\frac{\gamma}{s_{3}^{2}}+\frac{\delta}{s_{4}^{2}} .
$$

This potential is not Stäckel equivalent to a potential on Euclidean space.

Three of the four remaining systems can be obtained in the same way. However there is an alternative approach which enables us to obtain systems 2, 3, and 4 from 1 via well defined limiting processes. These are discussed elsewhere, e.g., Refs. 16 and 17, but we content ourselves with an example of how to obtain [211] from [1111]. If we make the transformations

$$
s_{1} \rightarrow \frac{1}{\sqrt{-\epsilon}} y_{1}, \quad s_{2} \rightarrow \frac{1}{\sqrt{\epsilon}}\left(y_{1}+\epsilon y_{2}\right), \quad \alpha \rightarrow-\sqrt{\beta} 2 \epsilon^{2}, \quad \beta \rightarrow \frac{\alpha}{\epsilon}-\frac{\beta}{2 \epsilon^{2}}
$$

then we deduce the relations

$$
y_{1}^{2}=-\frac{\left(u-e_{1}\right)\left(v-e_{1}\right)\left(w-e_{1}\right)}{\left(e_{1}-e_{2}\right)\left(e_{1}-e_{3}\right)}, \quad 2 y_{1} y_{2}=\frac{\partial}{\partial e_{1}} y_{1}^{2} .
$$

The coordinates on the sphere can be represented using the identifications $y_{1}=\left(s_{1}+i s_{2}\right) / \sqrt{2}, y_{2}$ $=\left(s_{1}-i s_{2}\right) / \sqrt{2}, y_{3}=s_{3}, y_{4}=s_{4}$ where $2 y_{1} y_{2}+y_{3}^{2}+y_{4}^{2}=s_{1}^{2}+s_{2}^{2}+s_{3}^{2}+s_{4}^{2}=1$. We then transform the potential according to $\alpha / s_{1}^{2}+\beta / s_{2}^{2}+\gamma / s_{3}^{2}+\delta / s_{4}^{2} \rightarrow \alpha / y_{1}^{2}+\beta y_{2} / y_{1}^{3}+\gamma / y_{3}^{2}+\delta / y_{4}^{2}$.

An exactly similar approach leads to the coordinates, constants of the motion and nondegenerate potential for the system [22]. Here the limit is taken in the form $e_{2}=e_{1}+\epsilon, e_{4}=e_{3}+\epsilon^{\prime}$ where $\epsilon, \epsilon^{\prime} \rightarrow 0$. For the system [31] we set $e_{2}=e_{1}+\epsilon, e_{3}=e_{1}+\epsilon^{\prime}$ and allow $\epsilon, \epsilon^{\prime} \rightarrow 0$, whereas for system [4] we set $e_{2}=e_{1}+\epsilon, e_{3}=e_{1}+\epsilon_{1}, e_{4}=e_{1}+\epsilon_{2}$ and allow $\epsilon, \epsilon_{1}, \epsilon_{2} \rightarrow 0$. In all cases except [4] the requirement that we have separation for all values of the parameters $e_{j}$ yields a set of six linearly independent second-order constants of the motion that can be verified to correspond to a nondegenerate superintegrable system. In the case [4] the constants of the motion do not depend on $e_{1}$ and we have only three independent symmetries. However, there is a unique potential that is obtained as the limit of the nondegenerate potential for case [1111]. By writing down the BertrandDarboux equations for this limit potential we can directly verify that it admits six linearly independent symmetries and is nondegenerate.

Theorem 8: Each of the five generic three-sphere separable systems determines a unique nondegenerate superintegrable system that permits separation simultaneously for all values of the parameters $e_{j}$. For each of these systems there is a basis of five (strongly) functionally independent and six linearly independent second order symmetries. In addition to system [VIII] above there are 
the following superintegrable systems (nondegenerate potential, followed by a basis of constants of the motion):

$\mathrm{I}^{\prime}$ [211] (Stäckel equivalent to the Euclidean superintegrable system [I])

$$
\begin{gathered}
V=\frac{\alpha_{1}}{\left(s_{1}+i s_{2}\right)^{2}}+\frac{\alpha_{2}\left(s_{1}-i s_{2}\right)}{\left(s_{1}+i s_{2}\right)^{3}}+\frac{\alpha_{3}}{s_{3}^{2}}+\frac{\alpha_{4}}{s_{4}^{2}} ; \\
\mathcal{S}_{0}=I_{12}^{2}+f_{0}, \quad \mathcal{S}_{1}=I_{34}^{2}+f_{1}, \quad \mathcal{S}_{2}=I_{13}^{2}+I_{23}^{2}+f_{2}, \\
\mathcal{S}_{3}=I_{14}^{2}+I_{24}^{2}+f_{3}, \quad \mathcal{S}_{4}=I_{13}\left(I_{13}+i I_{23}\right)+f_{4}, \quad \mathcal{S}_{5}=I_{14}\left(I_{14}+i I_{24}\right)+f_{5} .
\end{gathered}
$$

II' [22] (Stäckel equivalent to the Euclidean superintegrable system [II])

$$
\begin{gathered}
V=\frac{\alpha_{1}}{\left(s_{1}+i s_{2}\right)^{2}}+\frac{\alpha_{2}\left(s_{1}-i s_{2}\right)}{\left(s_{1}+i s_{2}\right)^{3}}+\frac{\alpha_{3}}{\left(s_{3}+i s_{4}\right)^{2}}+\frac{\alpha_{4}\left(s_{3}-i s_{4}\right)}{\left(s_{3}+i s_{4}\right)^{3}} ; \\
\mathcal{S}_{0}=I_{12}^{2}+f_{0}, \quad \mathcal{S}_{1}=I_{34}^{2}+f_{1}, \quad \mathcal{S}_{2}=I_{13}^{2}+I_{14}^{2}+I_{23}^{2}+I_{24}^{2}+f_{2}, \\
\mathcal{S}_{3}=I_{13}^{2}+I_{14}^{2}+i\left(I_{13} I_{23}+I_{14} I_{24}\right)+f_{3}, \quad \mathcal{S}_{4}=I_{13}^{2}+I_{23}^{2}+i\left(I_{13} I_{14}+I_{23} I_{24}\right)+f_{4}, \\
\mathcal{S}_{5}=I_{13}^{2}+I_{24}^{2}+i\left(I_{13} I_{14}+I_{13} I_{23}-I_{14} I_{24}-I_{23} I_{24}\right)-2 I_{13} I_{24}-I_{12} I_{34}+f_{5} .
\end{gathered}
$$

IV $^{\prime}$ [31] (Stäckel equivalent to the Euclidean superintegrable system [IV])

$$
\begin{gathered}
V=\frac{\alpha_{1}}{\left(s_{1}+i s_{2}\right)^{2}}+\frac{\alpha_{2} s_{3}}{\left(s_{1}+i s_{2}\right)^{3}}+\frac{\alpha_{3}\left(s_{1}^{2}+s_{2}^{2}-3 s_{3}^{2}\right)}{\left(s_{1}+i s_{2}\right)^{4}}+\frac{\alpha_{4}}{s_{4}^{2}} ; \\
\mathcal{S}_{0}=I_{12}^{2}+I_{13}^{2}+I_{23}^{2}+f_{0}, \quad \mathcal{S}_{1}=I_{14}^{2}+I_{24}^{2}+I_{34}^{2}+f_{1}, \quad \mathcal{S}_{2}=\left(I_{23}-i I_{13}\right)^{2}+f_{2}, \\
\mathcal{S}_{3}=I_{12}\left(I_{23}-i I_{13}\right)+f_{3}, \quad \mathcal{S}_{4}=I_{34}\left(I_{14}+i I_{24}\right)+f_{4}, \\
\mathcal{S}_{5}=I_{14} I_{34}-I_{12} I_{23}+i\left(I_{24} I_{34}+I_{12} I_{13}\right)+f_{5} .
\end{gathered}
$$

$\mathrm{VI}^{\prime}$ [4] (Stäckel equivalent to the Euclidean superintegrable system [VI])

$$
\begin{gathered}
V=\frac{\alpha_{1}}{\left(s_{1}+i s_{2}\right)^{2}}+\frac{\alpha_{2}\left(s_{3}+i s_{4}\right)}{\left(s_{1}+i s_{2}\right)^{3}}+\frac{\alpha_{3}\left[\left(s_{1}+i s_{2}\right)\left(s_{3}-i s_{4}\right)-\frac{3}{2}\left(s_{3}+i s_{4}\right)^{2}\right]}{\left(s_{1}+i s_{2}\right)^{4}} \\
+\frac{\alpha_{4}\left[\left(s_{1}+i s_{2}\right)\left(s_{1}^{2}+s_{2}^{2}-\frac{3}{2}\left(s_{3}^{2}+s_{4}^{2}\right)+\left(s_{3}+i s_{4}\right)^{3}\right]\right.}{\left(s_{1}+i s_{2}\right)^{5}} ; \\
\mathcal{S}_{0}=I_{12}^{2}+I_{13}^{2}+I_{14}^{2}+I_{23}^{2}+I_{24}^{2}+I_{34}^{2}+V, \\
\mathcal{S}_{1}=\left(I_{13}-I_{24}+i I_{23}+i I_{14}\right)^{2}+f_{1}, \\
\mathcal{S}_{2}=4\left(I_{23} I_{34}+I_{14} I_{34}+I_{13} I_{24}\right)+4 i\left(I_{24} I_{34}-I_{13} I_{34}\right)+2 i\left(I_{13} I_{23}-I_{14} I_{24}-I_{13} I_{14}+I_{23} I_{24}\right)-2 I_{12} I_{34}+I_{13}^{2} \\
+I_{24}^{2}-I_{14}^{2}-I_{23}^{2}+f_{2},
\end{gathered}
$$




$$
\begin{gathered}
\mathcal{S}_{3}=2\left(I_{12} I_{23}+I_{23} I_{34}-I_{12} I_{14}-I_{14} I_{34}\right)-2 i\left(I_{23} I_{24}+I_{13} I_{14}+I_{13} I_{34}+I_{24} I_{34}+I_{12} I_{24}+I_{12} I_{13}\right)-I_{13}^{2}+I_{24}^{2} \\
+I_{14}^{2}-I_{23}^{2}+f_{3}, \\
\mathcal{S}_{4}=\left(I_{13}-I_{24}+i I_{23}+i I_{14}\right)\left(I_{13}+I_{24}+i I_{23}-i I_{14}\right)+f_{4} \\
\mathcal{S}_{5}=\left(I_{13}-I_{24}+i I_{23}+i I_{14}\right)\left(I_{34}-I_{12}\right)+f_{5} .
\end{gathered}
$$

We also mention that the nongeneric superintegrable system on the three sphere with potential

$$
\mathbf{0 0}^{\prime} \quad V=\frac{\alpha}{\left(s_{1}+i s_{2}\right)^{2}}+\frac{\beta s_{3}}{\left(s_{1}+i s_{2}\right)^{3}}+\frac{\gamma s_{4}}{\left(s_{1}+i s_{2}\right)^{3}}+\frac{\delta\left(1-4 s_{3}^{2}-4 s_{4}^{2}\right)}{\left(s_{1}+i s_{2}\right)^{4}}
$$

is Stäckel equivalent to the Euclidean superintegrable system [00].

\section{F. Interbasis expansions for three-sphere systems}

In analogy with our treatment of Euclidean systems, to proceed with the classification of nondegenerate superintegrable systems on the three sphere we need to look more closely at the relationship between a standard basis of symmetries and the natural basis written in terms of the angular momentum generators $J_{\ell}, K_{\ell} \ell=1, \ldots, 3$. Then, near the regular point $\mathbf{T}$, i.e., $(x, y, z)$, we have a basis of "natural symmetries" $J_{1}=Y p_{Z}-Z p_{Y}, J_{2}=Z p_{X}-X p_{Z}, J_{3}=X p_{Y}-Y p_{X}, K_{1}=K_{X}, K_{2}$ $=K_{Y}, K_{3}=K_{Z}$. At the point itself we have $\left(1+r^{2} / 4\right) p_{u}=p_{X},\left(1+r^{2} / 4\right) p_{v}=p_{Y},\left(1+r^{2} / 4\right) p_{w}=p_{Z}$. Now suppose we have a three-sphere superintegrable system with nondegenerate potential. Then there will exist fifteen rational functions $A^{i j}[x, y, z], B^{i j}[x, y, z], C^{i j}[x, y, z]$, [with respect to the $(X, Y, Z)$ coordinates and restricted to the point $(X, Y, Z)=(0,0,0)$, that completely characterize the superintegrable system]. In particular, only ten of these, (23), are linearly independent, see relations (A2), and they are subject to the five quadratic conditions (A3) with $G(X, Y, Z)=\ln \lambda=-2 \ln (1$ $\left.+R^{2} / 4\right)$. These functions are related to the symmetries $\mathcal{S}=\sum a^{i j} p_{i} p_{j}+W$ via the conditions (A1). The second order basis symmetries at the regular point $\mathcal{S}_{\mathbf{x}_{0}}^{(\ell m)}(\mathbf{X})=\sum a_{(\ell m)}^{i j}(\mathbf{X}) p_{i} p_{j}+f_{(\ell m)}(\mathbf{X})$ take the form $\mathcal{S}_{\mathbf{x}_{0}}^{(\ell m)}(0,0,0)=p_{i} p_{j}+f_{(\ell m)}(0,0,0)$ when evaluated at the point. Thus we can expand each standard basis symmetry in a neighborhood of the point $(x, y, z)$ in terms of the natural basis at the point via

$$
\begin{aligned}
\mathcal{S}_{\mathbf{x}_{0}}^{(\ell m)}= & K_{\ell} K_{m}+\alpha_{3}^{(\ell m)} J_{1}^{2}+\alpha_{4}^{(\ell m)} J_{2}^{2}+\alpha_{5}^{(\ell m)} J_{3}^{2}+\alpha_{6}^{(\ell m)} K_{1} J_{1}+\alpha_{7}^{(\ell m)} K_{2} J_{2}+\alpha_{8}^{(\ell m)} K_{1} J_{2}+\alpha_{9}^{(\ell m)} K_{1} J_{3} \\
& +\alpha_{10}^{(\ell m)} K_{2} J_{1}+\alpha_{11}^{(\ell m)} K_{2} J_{3}+\alpha_{12}^{(\ell m)} K_{3} J_{1}+\alpha_{13}^{(\ell m)} K_{3} J_{2}+\alpha_{14}^{(\ell m)} J_{1} J_{2}+\alpha_{15}^{(\ell m)} J_{1} J_{3} \\
& +\alpha_{16}^{(\ell m)} J_{2} J_{3}+W^{(\ell m)}(\mathbf{X}),
\end{aligned}
$$

where the $\alpha_{k}^{(\ell m)}$ are constants in $X, Y, Z$ but rational functions of the parameters $x, y, z$ of the regular point. Here we are taking into account the identity $\Sigma_{h=1}^{3} K_{h} J_{h}=0$ and the fact that $K_{h}=p_{h}$ at the point $(X, Y, Z)=(0,0,0)$. Again, nondegenerate superintegrable system is uniquely determined by the ten numbers (23), and these numbers themselves are subject to five quadratic identities (A3). (Note that $G$ and all of its first and second derivatives vanish when $X=Y=Z=0$, except that $G_{i i}=-1, i=1,2,3$. Further, we can use relations (40) to express the derivatives of $V$ at the regular point with respect to the $(X, Y, Z)$ coordinates in terms of derivatives with respect to $(u, v, w)$. Thus the numbers (23) can be expressed as linear combinations of the corresponding numbers with respect to the $(u, v, w)$ coordinates.)

Although all of the expansion constants $\alpha_{k}^{(\ell m)}$ can be expressed in terms of these ten numbers, we shall restrict ourselves to expanding the two symmetries

$$
\begin{aligned}
\mathcal{S}_{\mathbf{x}_{0}}^{(12)}= & K_{1} K_{2}+\alpha_{3} J_{1}^{2}+\alpha_{4} J_{2}^{2}+\alpha_{5} J_{3}^{2}+\alpha_{6} K_{1} J_{1}+\alpha_{7} K_{2} J_{2}+\alpha_{8} K_{1} J_{2}+\alpha_{9} K_{1} J_{3}+\alpha_{10} K_{2} J_{1}+\alpha_{11} K_{2} J_{3} \\
& +\alpha_{12} K_{3} J_{1}+\alpha_{13} K_{3} J_{2}+\alpha_{14} J_{1} J_{2}+\alpha_{15} J_{1} J_{3}+\alpha_{16} J_{2} J_{3}+W^{(12)}(\mathbf{X})
\end{aligned}
$$




$$
\begin{aligned}
\mathcal{S}_{\mathbf{x}_{0}}^{(13)}= & K_{1} K_{3}+\alpha_{3}^{\prime} J_{1}^{2}+\alpha_{4}^{\prime} J_{2}^{2}+\alpha_{5}^{\prime} J_{3}^{2}+\alpha_{6}^{\prime} K_{1} J_{1}+\alpha_{7}^{\prime} K_{2} J_{2}+\alpha_{8}^{\prime} K_{1} J_{2}+\alpha_{9}^{\prime} K_{1} J_{3}+\alpha_{10}^{\prime} K_{2} J_{1}+\alpha_{11}^{\prime} K_{2} J_{3} \\
& +\alpha_{12}^{\prime} K_{3} J_{1}+\alpha_{13}^{\prime} K_{3} J_{2}+\alpha_{14}^{\prime} J_{1} J_{2}+\alpha_{15}^{\prime} J_{1} J_{3}+\alpha_{16}^{\prime} J_{2} J_{3}+W^{(13)}(\mathbf{X}) .
\end{aligned}
$$

(Here, $\alpha_{s}=\alpha_{s}^{(12)}, \alpha_{s}^{\prime}=\alpha_{s}^{(13)}$.) Since the six Bertrand-Darboux equations for these two symmetries have rank five, the symmetries completely determine the $A^{i j}, B^{i j}, C^{i j}$, hence the superintegrable system.

From (39)-(41) we have (with $J_{w}=u p_{v}-v p_{u}$ and cyclic permutations)

$$
\begin{aligned}
& J_{1}=\frac{1}{1+\frac{r^{2}}{4}}\left(\left(1+\frac{x^{2}}{4}-\frac{y^{2}}{4}-\frac{z^{2}}{4}\right) J_{u}+\frac{z x}{2} J_{w}+\frac{y x}{2} J_{v}-y K_{w}+z K_{v}\right), \\
& J_{2}=\frac{1}{1+\frac{r^{2}}{4}}\left(\left(1-\frac{x^{2}}{4}+\frac{y^{2}}{4}-\frac{z^{2}}{4}\right) J_{v}+\frac{x y}{2} J_{u}+\frac{z y}{2} J_{w}-z K_{u}+x K_{w}\right), \\
& J_{3}=\frac{1}{1+\frac{r^{2}}{4}}\left(\left(1-\frac{x^{2}}{4}-\frac{y^{2}}{4}+\frac{z^{2}}{4}\right) J_{w}+\frac{y z}{2} J_{v}+\frac{x z}{2} J_{u}-x K_{v}+y K_{u}\right), \\
& K_{1}=\frac{1}{1+\frac{r^{2}}{4}}\left(\left(1+\frac{x^{2}}{4}-\frac{y^{2}}{4}-\frac{z^{2}}{4}\right) K_{u}+\frac{y x}{2} K_{v}+\frac{z x}{2} K_{w}-y J_{w}+z J_{v}\right), \\
& K_{2}=\frac{1}{1+\frac{r^{2}}{4}}\left(\left(1-\frac{x^{2}}{4}+\frac{y^{2}}{4}-\frac{z^{2}}{4}\right) K_{v}+\frac{x y}{2} K_{u}+\frac{z y}{2} K_{w}-z J_{u}+x J_{w}\right), \\
& K_{3}=\frac{1}{1+\frac{r^{2}}{4}}\left(\left(1-\frac{x^{2}}{4}-\frac{y^{2}}{4}+\frac{z^{2}}{4}\right) K_{w}+\frac{x z}{2} K_{u}+\frac{y z}{2} K_{v}-x J_{v}+y J_{u}\right) .
\end{aligned}
$$

The inverse of these relations takes almost exactly the same form. Now, suppose we have a nondegenerate three-sphere superintegrable system with potential $V$, that is separable with respect to some orthogonal coordinates. (As every superintegrable system is multiseparable, we know that such coordinates exist.) By performing an Euclidean transformation, if necessary, we can assume that the separable coordinates are in some standard form determined by two constants of the motion in involution, $L_{1}=\sum a^{i j} p_{i} p_{j}+f_{1}, L_{2}=\sum b^{i j} p_{i} p_{j}+f_{2}$. Clearly, $L_{1}$ and $L_{2}$ lie in the sixdimensional space of second order symmetries for the superintegrable system. Thus, the quadratic form $a^{i j}$, for example, satisfies the three Bertrand-Darboux equations for potential $V$. Since $V$ is nondegenerate we can express the second derivatives $V_{j j}-V_{k k}$ and $V_{j k}$ with $j \neq k$ in the BertrandDarboux equations as linear combinations of the first derivatives $V_{h}$. Equating coefficients of $V_{1}, V_{2}, V_{3}$ separately in each of the three equations, we end up with nine linear conditions for the ten constants $A^{22}, \ldots, B^{23}$ at each regular point. If we choose the Cartesian-like coordinates $X, Y, Z$ that vanish at the regular point, then we obtain the same 18 conditions as in the Euclidean case. Indeed, the first derivatives $G_{i}$ all vanish at the regular point.

For the second symmetry there will be nine more such linear conditions with $a^{i j}$ replaced by $b^{i j}$. Thus we will have eighteen linear equations (not linearly independent) for the ten quantities $A^{22}, \ldots, B^{23}$.

The five fundamental quadratic identities (A3) are identical to those for the Euclidean case. This is because the only nonzero terms in the metric for the three sphere are $G_{i i}=-1$ and all such terms occur in the form $G_{i i}-G_{j j}=0$ in the five quadratic conditions.

Another source of conditions is obtained by writing the symmetry $L_{1}$ in terms of the standard basis: $a^{i j}(\mathbf{x})=\sum_{\ell \leqslant m} a^{\ell m}\left(\mathbf{x}_{0}\right) \mathcal{A}_{(\ell, m)}^{i j}(\mathbf{x})$, where $\mathcal{A}_{(\ell, m)}^{i j}$ is the quadratic form associated with the standard basis symmetry $\mathcal{S}^{(\ell, m)}$ at $\mathbf{x}_{0}$. Expanding both sides of this equation in terms of the natural 
basis we obtain linear and quadratic conditions on the ten basic quantities. In this case there is a difference between the Euclidean and three-sphere expressions. For example if we equate coefficients of the natural basis element $J_{1} J_{2}$ we find the quadratic conditions for $L_{1}$ and $L_{2}$

$$
-a_{12}^{33}\left(\mathbf{x}_{0}\right)=\sum_{\ell \leqslant m} a^{\ell m}\left(\mathbf{x}_{0}\right) \alpha_{14}^{(\ell m)}, \quad-b_{12}^{33}\left(\mathbf{x}_{0}\right)=\sum_{\ell \leqslant m} b^{\ell m}\left(\mathbf{x}_{0}\right) \alpha_{14}^{(\ell m)} .
$$

It is no longer true that $-a_{12}^{33}=2 a_{23}^{13}$ as in the Euclidean case. The expressions for the terms $\alpha_{14}^{(\ell m)}$ can be computed from the basic formulas (A1). They involve the terms $G_{i i}$ and differ from the Euclidean case. For example, from (A1) and formulas for the derivatives $\partial_{i} A^{j k}, \partial_{i} B^{j k}, \partial_{i} C^{j k}$ we can calculate $-a_{12}^{33}\left(\mathbf{x}_{0}\right)$ corresponding to the basis symmetry $\mathcal{S}^{(12)}$ and obtain

$$
\begin{aligned}
-3 \alpha_{14}^{(12)}= & \frac{1}{3} B^{22} C^{23}-\frac{2}{3}\left(C^{13}\right)^{2}-\frac{2}{3} C^{13} A^{22}+\frac{1}{6}\left(A^{23}\right)^{2}+\frac{1}{3} C^{33}\left(\frac{7}{2} B^{23}-2 C^{22}\right)+\frac{3}{2}-\frac{1}{2}\left(B^{33}\right)^{2}+\frac{5}{6} B^{33} B^{22} \\
& +\frac{5}{6}\left(B^{12}\right)^{2}+A^{33} B^{12}-\frac{1}{2}\left(B^{23}\right)^{2}+\frac{1}{6}\left(A^{12}\right)^{2}-\frac{1}{3} B^{33} A^{12}-\frac{1}{6} A^{22} A^{33}+\frac{1}{6}\left(A^{33}\right)^{2}-\frac{1}{6} C^{33} A^{13} .
\end{aligned}
$$

Though there are many other quadratic conditions for $L_{1}, L_{2}$ to belong to the symmetry algebra, we shall use only these two.

\section{G. Significance of generic three-sphere systems}

Suppose we have a nondegenerate superintegrable system that admits separation for some special choice of ellipsoidal coordinates [1111]. (Here we do not assume that the system separates for all values of the parameters $c, e_{1}, e_{2}, e_{3}, e_{4}$, but only for one value.) By performing an Euclidean transformation and a change of scale we can assume that the coordinates are in the standard form [1111] in our table and that $e_{1}=0, e_{2}=1, e_{3}=a$, and $e_{4}=b$ where $a, b$ are any fixed complex numbers such that $a b(a-1)(b-1)(b-a) \neq 0$. We follow the same method given before in the Euclidean case. We evaluate the $a^{i j}, b^{i j}$ at any regular point with coordinates $(x, y, z)$. Substituting these expressions into the eighteen linear conditions, with the help of MAPLE, we find that there are exactly seven independent linear conditions. Thus the ten quantities $A^{22}, \ldots, B^{23}$ can be expressed linearly in terms of three of these quantities. Substituting this result into the five fundamental quadratic identities (A3) we find that these identities yield exactly two solutions. Finally we substitute each of these solutions into (50) and find conditions that rule out one of these solutions. Thus only one solution exists and it must be the one that we already knew: System [VIII] that separates simultaneously for all choices of the parameters $e_{1}, \ldots, e_{4}$. What was far from obvious is the fact that no other nondegenerate superintegrable system separates for any special case of ellipsoidal coordinates on the three sphere.

Theorem 9: A three-sphere nondegenerate superintegrable system admits separation in a special case of the generic coordinates [1111], [211], [22], [31], or [4], respectively, if and only if it is equivalent via a complex rotation to system $[\mathrm{VII}],\left[\mathrm{I}^{\prime}\right],\left[I I^{\prime}\right],\left[I V^{\prime}\right]$, or $\left[\mathrm{VI}^{\prime}\right]$, respectively.

We have indicated the proof for coordinates [1111]. The other generic coordinates are Stäckel transforms of generic coordinates in Euclidean space so the proof for them follows immediately from Theorem 7.

\section{APPENDIX}

This is a list of some important results from Ref. 3. Using the nondegenerate potential condition and the Bertrand-Darboux equations we can solve for all of the first partial derivatives $a_{i}^{j k}$ of a quadratic symmetry to obtain the defining conditions [with $\lambda=\exp (G)]$

$$
\begin{aligned}
& a_{1}^{11}=-G_{1} a^{11}-G_{2} a^{12}-G_{3} a^{13}, \\
& a_{2}^{22}=-G_{1} a^{12}-G_{2} a^{22}-G_{3} a^{23}, \\
& a_{3}^{33}=-G_{1} a^{13}-G_{2} a^{23}-G_{3} a^{33},
\end{aligned}
$$




$$
\begin{aligned}
& 3 a_{1}^{12}=a^{12} A^{22}-\left(a^{22}-a^{11}\right) A^{12}-a^{23} A^{13}+a^{13} A^{23}+G_{2} a^{11}-2 G_{1} a^{12}-G_{2} a^{22}-G_{3} a^{23}, \\
& 3 a_{2}^{11}=-2 a^{12} A^{22}+2\left(a^{22}-a^{11}\right) A^{12}+2 a^{23} A^{13}-2 a^{13} A^{23}-2 G_{2} a^{11}+G_{1} a^{12}-G_{2} a^{22}-G_{3} a^{23}, \\
& 3 a_{3}^{13}=-a^{12} C^{23}+\left(a^{33}-a^{11}\right) C^{13}+a^{23} C^{12}-a^{13} C^{33}-G_{1} a^{11}-G_{2} a^{12}-2 G_{3} a^{13}+G_{1} a^{33}, \\
& 3 a_{1}^{33}=2 a^{12} C^{23}-2\left(a^{33}-a^{11}\right) C^{13}-2 a^{23} C^{12}+2 a^{13} C^{33}-G_{1} a^{11}-G_{2} a^{12}+G_{3} a^{13}-2 G_{1} a^{33}, \\
& 3 a_{2}^{23}=a^{23}\left(B^{33}-B^{22}\right)-\left(a^{33}-a^{22}\right) B^{23}-a^{13} B^{12}+a^{12} B^{13}-G_{1} a^{13}-2 G_{2} a^{23}-G_{3} a^{33}+G_{3} a^{22}, \\
& 3 a_{3}^{22}=-2 a^{23}\left(B^{33}-B^{22}\right)+2\left(a^{33}-a^{22}\right) B^{23}+2 a^{13} B^{12}-2 a^{12} B^{13}-G_{1} a^{13}+G_{2} a^{23}-G_{3} a^{33}-2 G_{3} a^{22}, \\
& 3 a_{1}^{13}=-a^{23} A^{12}+\left(a^{11}-a^{33}\right) A^{13}+a^{13} A^{33}+a^{12} A^{23}-2 G_{1} a^{13}-G_{2} a^{23}-G_{3} a^{33}+G_{3} a^{11}, \\
& 3 a_{3}^{11}=2 a^{23} A^{12}+2\left(a^{33}-a^{11}\right) A^{13}-2 a^{13} A^{33}-2 a^{12} A^{23}+G_{1} a^{13}-G_{2} a^{23}-G_{3} a^{33}-2 G_{3} a^{11}, \\
& 3 a_{2}^{33}=-2 a^{13} C^{12}+2\left(a^{22}-a^{33}\right) C^{23}+2 a^{12} C^{13}-2 a^{23}\left(C^{22}-C^{33}\right)-G_{1} a^{12}-G_{2} a^{22}+G_{3} a^{23}-2 G_{2} a^{33}, \\
& 3 a_{3}^{23}=a^{13} C^{12}-\left(a^{22}-a^{33}\right) C^{23}-a^{12} C^{13}-a^{23}\left(C^{33}-C^{22}\right)-G_{1} a^{12}-G_{2} a^{22}-2 G_{3} a^{23}+G_{2} a^{33}, \\
& 3 a_{2}^{12}=-a^{13} B^{23}+\left(a^{22}-a^{11}\right) B^{12}-a^{12} B^{22}+a^{23} B^{13}-G_{1} a^{11}-2 G_{2} a^{12}-G_{3} a^{13}+G_{1} a^{22}, \\
& 3 a_{1}^{22}=2 a^{13} B^{23}-2\left(a^{22}-a^{11}\right) B^{12}+2 a^{12} B^{22}-2 a^{23} B^{13}-G_{1} a^{11}+G_{2} a^{12}-G_{3} a^{13}-2 G_{1} a^{22}, \\
& 3 a_{1}^{23}=a^{12}\left(B^{23}+C^{22}\right)+a^{11}\left(B^{13}+C^{12}\right)-a^{22} C^{12}-a^{33} B^{13}+a^{13}\left(B^{33}+C^{23}\right)-a^{23}\left(C^{13}+B^{12}\right) \\
& -2 G_{1} a^{23}+G_{2} a^{13}+G_{3} a^{12} \text {. } \\
& 3 a_{3}^{12}=a^{12}\left(-2 B^{23}+C^{22}\right)+a^{11}\left(C^{12}-2 B^{13}\right)-a^{22} C^{12}+2 a^{33} B^{13}+a^{13}\left(-2 B^{33}+C^{23}\right) \\
& +a^{23}\left(-C^{13}+2 B^{12}\right)-2 G_{3} a^{12}+G_{2} a^{13}+G_{1} a^{23} \text {. } \\
& 3 a_{2}^{13}=a^{12}\left(B^{23}-2 C^{22}\right)+a^{11}\left(B^{13}-2 C^{12}\right)+2 a^{22} C^{12}-a^{33} B^{13}+a^{13}\left(B^{33}-2 C^{23}\right)+a^{23}\left(2 C^{13}-B^{12}\right) \\
& -2 G_{2} a^{13}+G_{1} a^{23}+G_{3} a^{12},
\end{aligned}
$$

plus the linear relations

$$
\begin{gathered}
A^{23}=B^{13}=C^{12}, \quad B^{23}-A^{31}-C^{22}=0, \\
B^{12}-A^{22}+A^{33}-C^{13}=0, \quad B^{33}+A^{12}-C^{23}=0 .
\end{gathered}
$$

Using the linear relations we can express $C^{12}, C^{13}, C^{22}, C^{23}$, and $B^{13}$ in terms of the remaining ten functions. Finally, requiring that the integrability conditions for (A1) hold identically, we obtain exactly five quadratic identities for the ten independent functions:

$$
\begin{aligned}
-A^{23} B^{23}-A^{12} A^{23}+A^{13} B^{12}+B^{22} A^{23}+B^{23} A^{33}+\frac{1}{2} A^{22} G_{3}-\frac{1}{2} A^{33} G_{3}-\frac{1}{2} B^{12} G_{3}-\frac{1}{2} G_{1} G_{3}-\frac{1}{2} A^{13} G_{1} \\
+\frac{3}{2} G_{13}-\frac{1}{2} A^{23} G_{2}-A^{22} B^{23}=0,
\end{aligned}
$$




$$
\begin{aligned}
& \left(A^{33}\right)^{2}+B^{12} A^{33}-A^{33} A^{22}-B^{33} A^{12}-C^{33} A^{13}+B^{22} A^{12}-B^{12} A^{22}+A^{13} B^{23}-\left(A^{12}\right)^{2}+\frac{3}{2} G_{22}-\frac{1}{2} G_{y}^{2} \\
& -\frac{3}{2} G_{33}+\frac{1}{2} A^{13} G_{3}+\frac{1}{2} B^{33} G_{2}-\frac{1}{2} A^{22} G_{1}+\frac{1}{2} A^{33} G_{1}-\frac{1}{2} B^{23} G_{3}-\frac{1}{2} B^{22} G_{2}+\frac{1}{2} C^{33} G_{3}+\frac{1}{2}\left(G_{3}\right)^{2}=0 \\
& -\left(B^{33}\right)^{2}-B^{33} A^{12}+B^{33} B^{22}+B^{12} A^{33}+B^{23} C^{33}-\left(B^{23}\right)^{2}+\left(B^{12}\right)^{2}+\frac{1}{2}\left(G_{1}\right)^{2}-\frac{3}{2} G_{11}+\frac{3}{2} G_{33} \\
& -\frac{1}{2} B^{33} G_{2}-\frac{1}{2} A^{33} G_{1}-\frac{1}{2}\left(G_{3}\right)^{2}-\frac{1}{2} C^{33} G_{3}=0, \\
& -B^{12} A^{23}-A^{33} A^{23}+A^{13} B^{33}+A^{12} B^{23}+\frac{3}{2} G_{23}-\frac{1}{2} A^{23} G_{1}-\frac{1}{2} A^{12} G_{3}-\frac{1}{2} B^{23} G_{2}-\frac{1}{2} G_{2} G_{3} \\
& -\frac{1}{2} B^{33} G_{3}=0 \\
& A^{12} B^{12}+C^{33} A^{23}-A^{23} B^{23}+B^{33} A^{22}-B^{33} A^{33}+\frac{3}{2} G_{12}-\frac{1}{2} G_{1} G_{2}-\frac{1}{2} A^{12} G_{1}-\frac{1}{2} B^{12} G_{2}-\frac{1}{2} A^{23} G_{3}=0
\end{aligned}
$$

${ }^{1}$ E. G. Kalnins, J. M. Kress, and W. Miller, Jr., J. Math. Phys. 46, 053509 (2005).

${ }^{2}$ E. G. Kalnins, J. M. Kress, and W. Miller, Jr., J. Math. Phys. 46, 053510 (2005).

${ }^{3}$ E. G. Kalnins, J. M. Kress, and W. Miller, Jr., J. Math. Phys. 46, 103507 (2005).

${ }^{4}$ F. Calogero, J. Math. Phys. 10, 2191 (1969).

${ }^{5}$ C. Grosche, G. S. Pogosyan, and A. N. Sissakian, Fortschr. Phys. 43, 453 (1995).

${ }^{6}$ S. R. Wojciechowski, Phys. Lett. 95A, 279 (1983).

${ }^{7}$ A. Ballesteros, F. Herranz, M. Santander, and T. Sanz-Gil, J. Phys. A 36, L93 (2003).

${ }^{8}$ M. Rodriguez and P. Winternitz, J. Math. Phys. 43, 1309 (2002).

${ }^{9}$ L. G. Mardoyan, G. S. Pogosyan, A. N. Sissakian, and V. M. Ter-Antonyan, Nuovo Cimento Soc. Ital. Fis., B 88, 43 (1985).

${ }^{10}$ Superintegrability in Classical and Quantum Systems, edited by P. Tempesta, P. Winternitz, W. Miller, and G. Pogosyan (AMS, Providence, Rhode Island, 2005), Vol. 37.

${ }^{11}$ G. S. Pogosyan and P. Winternitz, J. Math. Phys. 43, 3387 (2002).

${ }^{12}$ P. Letourneau and L. Vinet, Ann. Phys. 243, 144 (1995).

${ }^{13}$ M. Blaszak and A. Sergyeyev, J. Phys. A 38, L1 (2005).

${ }^{14}$ C. P. Boyer, E. G. Kalnins, and W. Miller, SIAM J. Math. Anal. 17, 778 (1986).

${ }^{15}$ J. Hietarinta, B. Grammaticos, B. Dorizzi, and A. Ramani, Phys. Rev. Lett. 53, 1707 (1984).

${ }^{16}$ M. Bôcher, Über die Riehenentwickelungen der Potentialtheorie. Teubner, Leipzig, 1894 (in German).

${ }^{17}$ E. G. Kalnins, W. Miller, and G. K. Reid, Proc. R. Soc. London, Ser. A 394, 183 (1984).

${ }^{18}$ C. P. Boyer, E. G. Kalnins, and W. Miller, J. Math. Phys. 19, 200 (1978).

${ }^{19}$ L. P. Eisenhart, Riemannian Geometry, 5th ed. (Princeton University Press, Princeton, 1964).

${ }^{20}$ E. G. Kalnins, Separation of Variables for Riemannian Spaces of Constant Curvature, Pitman, Monographs and Surveys in Pure and Applied Mathematics Vol. 28 (Longman, Essex, England, 1986), 184-208.

${ }^{21}$ W. Miller, Jr., Symmetries and Non-linear Phenomena (World Scientific, Singapore 1988), pp. 188-221.

${ }^{22}$ E. G. Kalnins and W. Miller, Jr., SIAM J. Math. Anal. 9, 12 (1978). 